\title{
Data Collection in Studies on Internet of Things (IoT), Wireless Sensor Networks (WSNs), and Sensor Cloud (SC): Similarities and Differences
}

\author{
IHSAN ALI \\ Department of Computer System \& Technology, Faculty of Computer Science \& Information Technology, University of Malaya, Malaysia \\ Corresponding authors: Ihsan Ali(ihsanalichd@siswa.um.edu.my)
}

This research work was partially supported by the Faculty of Computer Science and Information Technology, University of Malaya under Postgraduate Research Grant (PG035-2016A).

\begin{abstract}
Data collection is an essential part of sensor devices, particularly in such technologies like Internet of Things (IoT), wireless sensor networks (WSN), and sensor cloud (SC). In recent years, various literature had been published in these research areas to propose different models, architectures, and contributions in the domains. Due to the importance of efficient data collection regarding reducing energy consumption, latency, network lifetime, and general cost, a momentous literature volume has been published to facilitate data collection. Hence, review studies have been conducted on data collection in these domains in isolation. However, a lack of comprehensive review collectively identifies and analyzes the differences and similarities among the data collection proposals in IoT, WSN, and SC. The main objective of this research is to conduct a comprehensive survey to explore the current state, use cases, contributions, performance measures, evaluation measures, and architecture in the IoT, WSN, and SC research domains. The findings indicate that studies on data collection in IoT, WSN, and SC are relatively consistent with stable output in the last five years. Nine novel contributions are found with models, algorithms, and frameworks being the most utilized by the selected studies. In conclusion, key research challenges and future research directions have been identified and discussed.
\end{abstract}

INDEX TERMS Data Collection, WSNs, Sensor Cloud, Architecture, IoT

\section{INTRODUCTION}

Wireless sensor networks (WSNs) are the most significant component of the Internet of things (IoTs) [1]. IoT and WSNs have been implemented in many applications, such as transport system [2]building automation [3], agriculture [4], [5], health monitoring [6] [7] and so on. However, the battery capacity, storage capability, and data transmission rate of the sensors are known to be insufficient, which can affect the network lifetime. The study of Piyare et al. [8] proposed extensible and flexible architecture for integrating WSN with the cloud to improve the transmission speed and network lifetime. The integration of WSN and cloud in sensor cloud (SC) not only improves WSN lifetime but also service quality, computation latency, and energy consumption [9]. The main objective of the SC is that it allows a single WSN to generate sensing services to various applications concur- rently [10]. Over the past decade, numerous studies have proposed various solutions in these research domains. These studies contributed state-of-art solution proposals, evaluation mechanisms, performance metrics, and distinct architectures in IoT, WSN, and SC [11]-[13]. However, to date, no study has yet to comprehensively examine the differences and similarities among IoT, WSN, and SC. Hence, to fill this research gap, the current study conducted a comprehensive survey to explore the current state, use cases, contributions, performance measures, evaluation measures, and architecture in the IoT, WSN, and SC research domains. A thorough and comprehensive search process was conducted to ensure that relevant studies on the three selected domains in the last decade (2010-2020) were identified and retrieved. This survey also presented results based on the selected studies' (SS) characteristics, the proposed contributions, the use cases 
1. Introduction
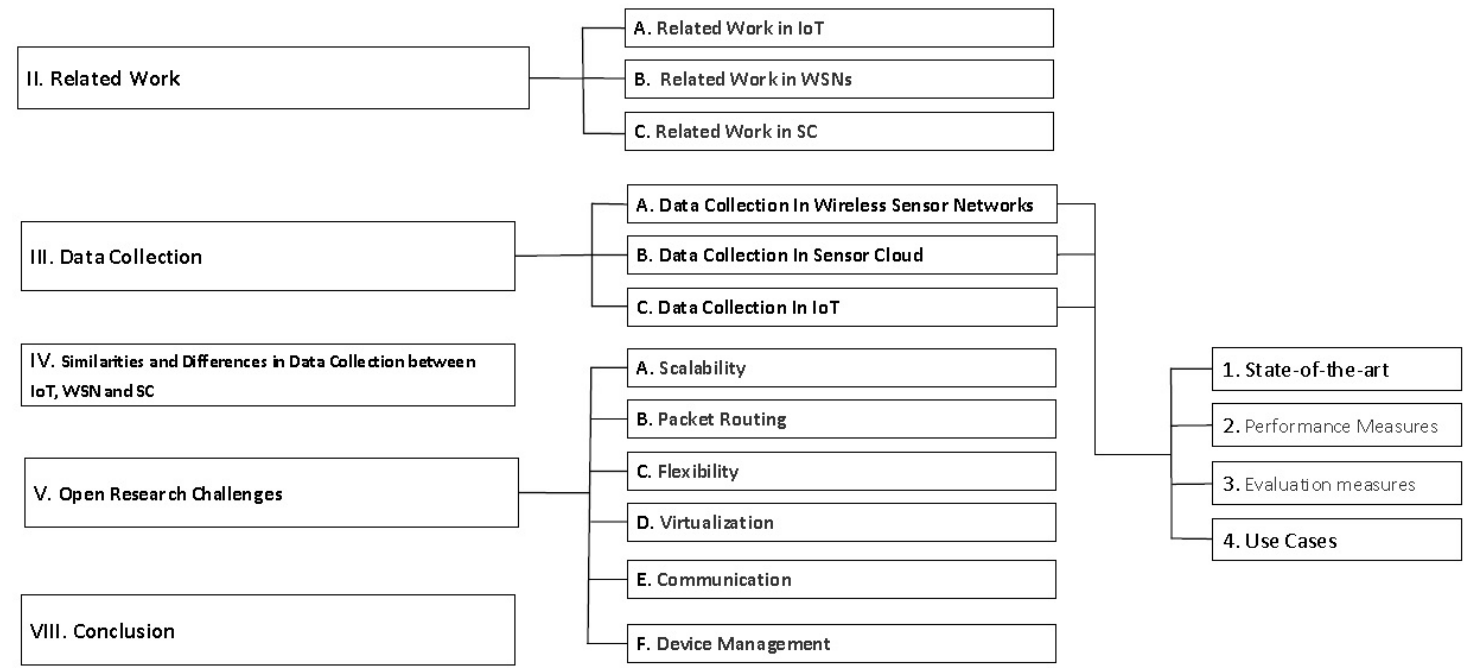

FIGURE 1. Overview of the Paper

of the SS, the evaluation mechanisms used by the SS, the performance measures of the studies, and the architectures used. The contributions provided by this study are as follows:

- A comprehensive review of the three selected domains: IoT, WSN, and SC.

- Analysis and synthesis of current works in this research domain.

- Finding the similarities and the differences between the three selected domains.

- Identifying existing research challenges and areas that need attention from the research community in this domain.

The rest of the article is organized as follows: the related works are assessed and presented in Section II, Section III describes the data collection processes in the selected domains, Section IV presents and discusses the similarities and differences, Section V outlines the open research challenges, and finally, the study is concluded in Section VI.Figure 1 represent a road map of the paper. All the abbrivation used in this paper are given in table 8 .

\section{LITERATURE REVIEW}

This section highlights and discusses the existing review and survey studies in the IoT, WSN, and SC research domains. The research fields are classified based on the sub-sections described below. Presenting and discussing these studies can help in solidifying the importance of conducting this kind of survey.

\section{A. RELATED WORK IN IOT}

$\mathrm{Xu}$ et al. presented a survey of IoT in industries [14] in which they systematically summarized and highlighted the current state of IoT in industries. The study focused on identifying the context and classifying the existing studies in IoT in an industrial setting. Although the study highlighted the challenges and opportunities in IoT for novel and veteran researchers in this domain, only related articles published in the web of knowledge database from 2009-2013 were used in the study. Meanwhile, another study reviewed the existing technologies of IoT and sensor networks [15] in which the authors defined a six-layered architecture of IoT and highlighted related issues and challenges. However, the study limited its scope to architecture, issues, and challenges. In Fortino et al., a comparative study was presented wherein the authors proposed a comparison based on IoT layer's vital characteristics and the kind of architecture being used [16]. Similarly, Ferrag et al. surveyed existing blockchain protocols used in IoT networks and highlighted the existing studies that focused on blockchain and further described the identified blockchain [17]. That study also discussed blockchain application domains in IoT as well as provided a taxonomy and state-of-the-art methods used in the reviewed studies for securing and privacy-preservation of blockchain technologies. Additionally, Luong et al. surveyed data collection and wireless communication in IoT by utilizing pricing models and economic analysis [18]. The authors reviewed the existing literature on pricing models, applications, and economic analysis for data collection and wireless communication in IoT. Furthermore, the survey highlighted research problems with future research directions for applying pricing and economic to IoT.

\section{B. RELATED WORK IN WSNS}

In Rawat et al., a survey on potential synergies and recent developments in WSN was presented [19]. The survey described the synergy between WSN and other technologies 
as well as presented the existing challenges and issues of WSN and future research directions. The survey conducted by Pantazis et al. discussed energy-efficient routing protocols in WSNs and highlighted extensive issues in each of the routing protocols [20]. The study's classified energyefficient routing protocols included topology-based reliable routing, network structure, and communication model. The protocols' weaknesses and strengths were also presented to enable researchers to choose an appropriate protocol to suit their needs. However, the paper did not discuss significant performance metrics in WSN, such as reliability, scalability, efficiency, and so on. Oliviera and Rodriques presented a survey on WSN solutions used in monitoring environmental applications [21]. The authors critically analyzed related projects on environmental monitoring with real deployments and highlighted future studies' challenges. Hence, the paper focused on monitoring environmental application use cases. In another study, Yang et al. conducted a survey describing the IoT security and privacy issues [22]. The study started by presenting the solutions to the IoT device limitations and then highlighting current IoT attacks' taxonomy. The authors further reviewed the IoT access control schemes, authentication, and architectures of the existing studies. However, only limited solutions were reviewed to address the study's challenges. Hodge et al. surveyed WSN technology in the railway industry [23], focusing on practical engineering solutions where sensor devices were used. However, the paper only examined the railway industry use case. Similarly, Das et al. presented a survey study on WSN virtualization [24], which provided a forum where multiple applications can exist in a single framework of sensor infrastructure, thus minimizing deployment cost, the number of sensors, and so on, of sensor infrastructure. Another study surveyed data collection in WSNs, provided a standard classification of WSN architectures, and discussed the process data collection and the current problems [25]. The study also described other important data collection processes, such as performance measures and evaluation mechanisms not discussed in other studies. In a survey by [26], the authors discussed the existing works on WSNs and WSN packages, such as design features, applications, and lifetime prediction models. Another study presented a comprehensive survey of existing studies concentrating on using WSN for structural health monitoring [27]. The authors focused on WSN telecommunication in the existing structural health monitoring (SHM) studies and highlighted the challenges and future trends in the application of SHM to WSNs.

\section{RELATED WORK IN SENSOR CLOUD}

In a study by Alamri et al. [28], the authors presented a comprehensive study on SC infrastructure. They reviewed SC constraints, including architecture, applications, use cases, and definitions. That work also highlighted the challenges, proposed solutions, techniques, and directions for future research. However, the paper neither discussed the performance metric and system models adopted in the SC nor provided a detailed evaluation of the facets considered . [29] used cloud computing to survey typical SC network applications and reviewed how cloud computing applications were combined with WSNs in use cases like environmental monitoring, weather prediction and forecasting, healthcare, transportation business, and military application. Notably, the paper did not discuss the significant SC data collection process. The survey on SC studies conducted by Ansari et al. [28] highlighted the architecture, definitions, and processing time of SC and the layered approach of SC. Dwivedi et al. [30] presented a survey study that described the security attacks on SC. The authors also discussed the existing studies' defence mechanism to protect SC from security attacks. Although the literature indicates a significant number of surveys in IoT, WSN, and SC, to the best of our knowledge, existing works dedicated to surveying the specific relations among the three domains (IoT, WSN, and SC) are nonexistent. Therefore, this paper surveyed to explore the stateof-the-art, use cases, contributions, performance measures, evaluation measures, and architecture in the IoT, WSN, and $\mathrm{SC}$ research domains. Hence, we extracted information on various evaluation measures, architectures, and use cases in the selected domains. Then, the differences and similarities among IoT, WSN, and SC were identified.

\section{DATA COLLECTION}

In this section, studies on data collection of the three facets of this research (data collection in IoT, WSN, and SC) are presented and critically discussed in detail.

\section{A. DATA COLLECTION IN THE INTERNET OF THINGS}

This subsection outlines the detailed discussion and analysis of data collection related to IoT. Details on the current state, proposals, performance measures, evaluation measures, and use cases were outlined and discussed.

\section{1) State-of-the-art}

In a study by $\mathrm{Hu}$, the author proposed a software-defined Industrial IoT (IIoT) architecture to provide a solution to holistic IIoT system challenges [31]. The proposed architecture was formulated based on novel networking technologies, such as software-defined networking (SDN) and constrained application protocol (CoAP). Rao et al. proposed a prototype model that describes how IoT and cloud computing collaboratively works and address big data issues [32]. In a study by Savale et al., the authors proposed a framework that connects IoT with agriculture to provide links between farms with agronomists, thus increasing agricultural product harvests [33]. The study provides a comprehensive system designed to achieve higher precision in agricultural production. Sheng et al. proposed a lightweight approach that allows for the device management of wireless sensor devices [34]. They were inspired by the recent development of the IPv6 protocol, which they adopted in their proposed approach. The study developed a prototype to ascertain the proposed approach's efficiency in managing wireless sensor devices, thus facilitat- 
ing IoT development. Feng et al. identified the feasible, best, and practical wireless communication technologies applied in precision agriculture [4] . They also conducted experimental test agricultural scenario analyses and detected three WSN architectures. They then proposed and developed a novel NB-IoT technology-based precision agricultural system. Kumar and Ramudu developed a comprehensive system to aid precision agriculture [35]. The proposed approach provides instant output regarding the crops and land to enable farmers to make the best agricultural decisions. Kamalinejad et al. proposed a unit for wireless energy harvesting in IoT called WEH-IoT [36]. They further analyzed the WEH-IoT lifetime based on two scenarios in IoT systems. The simulation results show that the lifetime improved by using the proposed techniques. Kirichek et al. [37] proposed a model that provides a comprehensive review of the peculiarities of IoT devices, applications, and protocols. Another study proposed a scheme for cognitive radio sensor networks (CRSNs) [38]. In the CRSN architecture, the sensor nodes can access the harvested energy from ambient radio-frequency sources. The authors then introduced a new energy management scheme, which allows sensor nodes to achieve energy harvesting and cluster head selection algorithm. The study also conducted a simulation experiment to ascertain the scheme's effectiveness over compared schemes. Ilyas et al. introduced a trust-based energy-efficient routing protocol (TBEERP) that aims to overcome IoT challenges to extend network lifetime and decrease latency [39]. The study was evaluated based on a comparison with existing algorithms. The simulation results revealed a good performance when equated with existing algorithms. Meanwhile, Alam et al. introduced an IoT framework to support connected objects sensor reasoning and processing by providing a semantic overlay of the primary IoT cloud [40]. They then validated the framework through prototype implementation on a real-life battery driven motor. Chen et al. tailored a system that can promote the popularity of narrowband-IoT [41]. The tailored system consisted of basic components integrated. Huh et al. developed a novel system using Ethereum, a blockchain computing platform to control and configure IoT devices. They used Raspberry Pis for the proposed IoT system simulation. Orsino et al. proposed a device-to-device-based approach [42], which they evaluated using simulation analysis to ascertain its performance in broad scenarios, thus identifying attainable gains in terms of energy efficiency-based IoT data collection. Li et al. developed an optimal computing resource allocationbased algorithm [43]. The results of the theoretical analysis and simulations of the proposed algorithm show that it is efficient and can provide good business strategies for consumers and retailers in the field of computing resource. Siboni et al. introduced an innovative framework for the IoT devices [44]. The proposed framework handles security and privacy threats in the IoT devices. Ahmed et al. investigated and discussed the state-of-the-art research conducted in the domain of IoT-based smart environments [45]. The study highlighted the significance of IoT and smart environment integration and further detailed the taxonomy of IoT in such environments. It also conducted a case study in IoT-based smart environments. Antao et al. highlighted the conditions for Cyber Physical Production Systems and Industrial IoT by proposing a requirements list for validation [46]. Yang et al. highlighted the research issues of IoT-enabled manufacturing [47] IoT's core technologies to be used in addressing the manufacturing challenges are radio frequency identification, WSN, cloud computing, and Big Data. Soldatos and Serrano proposed IoT cloud environment design principles and framework to converge IoT Infrastructure with cloud-based computing models and applications into the cloud [48]. The study analyzed state-of-the-art results and efforts concerning cloud computing and IoT convergence. The authors also presented the framework building block, which emphasizes the formation of IoT services based on Internet-connected objects. The study by Atzori et al. presented opportunities obtained from the incorporation of networking concepts into the IoT and found the stages of increasing levels of social involvement [49]. Zimmermann et al. investigated mechanisms for IoT decision-controlled architectures and microservices by evolving reference models and architecture [50]. The Ray study presented a novel H3IoT architectural framework to monitor the health conditions of elderly individuals [6]. The framework showed good performance in terms of its utilization for wellness and decreasing the present healthcare system's strain points. Brooks et al. described an IoT component-based software architecture wherein the proxies accessory interact with one another [51]. Sisinni et al. highlighted the concepts of IoT, industrial IoT, and industry 4.0; described the benefit generated by this paradigm shift; and discussed the related issues [52]. Qu et al. presented a summary of privacy issues and possible attacks based on newly designed IoT features [53]. They conducted three case studies to classify the schemes. Chamoso et al. reviewed IoT studies on the so-called "smart cities," provided an analysis of the concept and current platforms, and proposed a model for designing a smart city architecture [7]. Kovatsch et al. presented a Cloud/IoT architecture based on the Constrained Application Protocol (CoAP), typically used in low-cost IoT devices [54]. The study systematically evaluates the system architecture and protocol performance in cloud environments. The evaluation results show that the CoAP framework provides higher throughput than high-performance. Wang et al. presented an ontology for the IoT domain by incorporating models during the modelling process on the IoT, providing support for IoT task, and exploiting the existing efforts' synergy [55]. Korzun et al. highlighted their experiences while using smart space applications [56]. They performed experiments to estimate the response time of the Smart-M3 platform and determined its applicability in current computing environments. Liu et al. proposed LightChain, which is suitable for power-constrained industrial IoT [57]. An experiment conducted showed that LightChain decreased the computational cost and sped up the block generation process. In another study, a technique called FaBric blockchain- 
based data transmission was proposed for industrial IoT data transmission [58]. Experiments showed the high reliability and security of the optimized FaBric power data storage and transmission. Liu et al. adopted the use of unmanned aerial vehicle (UAV) to assist in emergency communications in a heterogeneous IoT (Het-IoT) environment [59]. Similarly, the authors proposed nonorthogonal multiple access (NOMA) and a multi-objective resource allocation (MORA) scheme to handle the communications of the remaining users and IoT devices for the UAV-assisted Het-IoT. Their simulation results confirmed that the scheme provides efficient performances for the users and the devices. Chen et al. proposed an online and polynomial-time complexity algorithm. Experimental results showed the effectiveness of the proposed algorithm in reducing energy consumption. Alamed presents a middleware framework that incorporated the IoT, Fog, and Blockchain technologies [60]. Table 1 presents the details of the data collection studies.

Figure 2 shows the respective publication channels identified from the studies on data collection in IoT. Hence, the number of studies for data collection in IoT is also given in the figure. We have identified three publication channels, which are journals, conferences, and magazines, with journals having the highest number of studies (16), followed by conferences (12), and magazines (1).

\section{2) Performance Measures}

In this section, the SS's performance measures are outlined and presented. These performance measures are also presented in Table 2 with the respective studies that considered them. However, only the performance measures of data collection studies on IoT are given in this subsection. We have identified 19 performance measures used by the SS. We found that the most considered performance measures are network lifetime, performance, and privacy. However, we observed that 12 studies did not clearly outline which performance measures they utilized in their respective studies; hence, they were categorized as "Nil."

\section{3) Evaluation measures}

From Table 2, the reader can observe that the evaluation measures for each study are provided. For the analysis in this section, we have identified four evaluation measures: Experiment - adopted by 5 studies, Simulation - adopted by 11 studies, Case study - adopted by 5 studies, and Hybrid (the combination of two or more evaluation measures) adopted by 2 studies. Figure 3 highlights each evaluation measure's detailed breakdown with respect to the years of use in line with the respective studies. For Simulation, which is the most commonly used by studies on data collection for IoT, 2019 was the most active year, with four studies utilizing this method .

\section{4) Use Cases}

This section presents the use cases of data collection in IoT. From the SS, we identified several use cases, namely, agriculture, augmented reality and environment monitoring, smart environment, manufacturing and smart cities, trade, health, mechanical sector, software engineering, collaborative work, tourism, and mathematical analysis. In our subcategorization, we categorize discussions on other use cases as "others" because only a few studies have used such cases. Agriculture Data collection in agriculture has become imperative in today's farming, where sensor technologies are utilized in the process. Hence, the sense data are to be collected effectively. Results show that most of the studies reviewed investigated the IoT in an agricultural setting [4] [32] [33] [35]. This is because IoT is used to monitor and select crop growth and provide irrigation decision support, among others, [32] illustrated data collection in IoT using software and applications for agriculture. Recent studies [4] [35] [33] have investigated wireless communication technologies on IoT data collection to improve agricultural precision. Smart environment Smart environments have become very popular in recent years, with ever-increasing technologies being deployed to improve technological and data accessibility awareness. Looking at our environment, IoT and sensing devices are deployed at a large scale without noticing. Hence, with this large deployment of sensing devices, finding methods of collecting key data from the sensing devices is crucial. From our review, we have identified a few studies that worked on data collection for smart environments. For instance, some studies proposed energy-efficient IoT data collection in smart cities by exploiting various communication technologies, while others studied the issue holistically [45] [49] [39] [42] [31] [42] [34]. Manufacturing We identified several studies that utilized IoT in data collection for manufacturing [52] [22]. Due to the importance of IoT in the manufacturing environment, various data collection mechanisms are needed, as the precise collection of data in this field is imperative. Hence, we recommend the research community to focus on this area due to its importance. Health care The health care sector is another important area in which a great deal of data is generated with increased IoT technology use. Hence, collecting precise data for patients' diagnosis is very important. One study utilized IoT in healthcare and smart environment [7], while another [6] concentrated on building a health-related IoT framework. Others The rest of the studies [40] [48] [22] [50] used IoT in the mechanical sector. [4] used IoT in trade, Chen et al. [41] used the technology in academic research, [45] and Wang et al. [55] used it in software engineering, and finally, [41] used it in mathematical analysis.

\section{B. DATA COLLECTION IN WIRELESS SENSOR NETWORK}

This subsection outline the detailed discussion and analysis of data collection with respect to WSNs. Several aspects, such as the state-of-the-art, proposals, performance measures, evaluation measures, and use cases, are outlined and discussed in this section. 


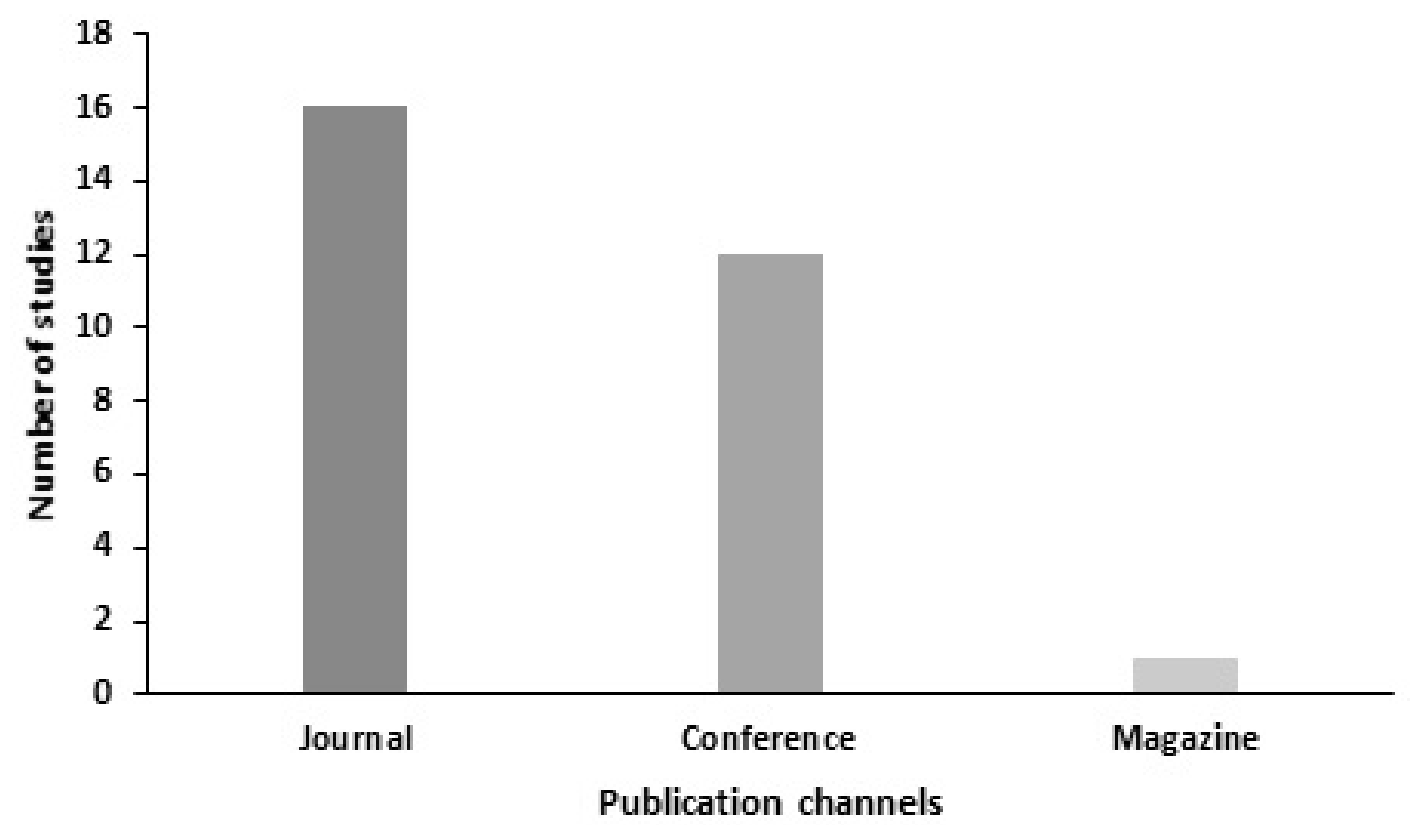

FIGURE 2. Analysis of the publication channels for DC studies in loT
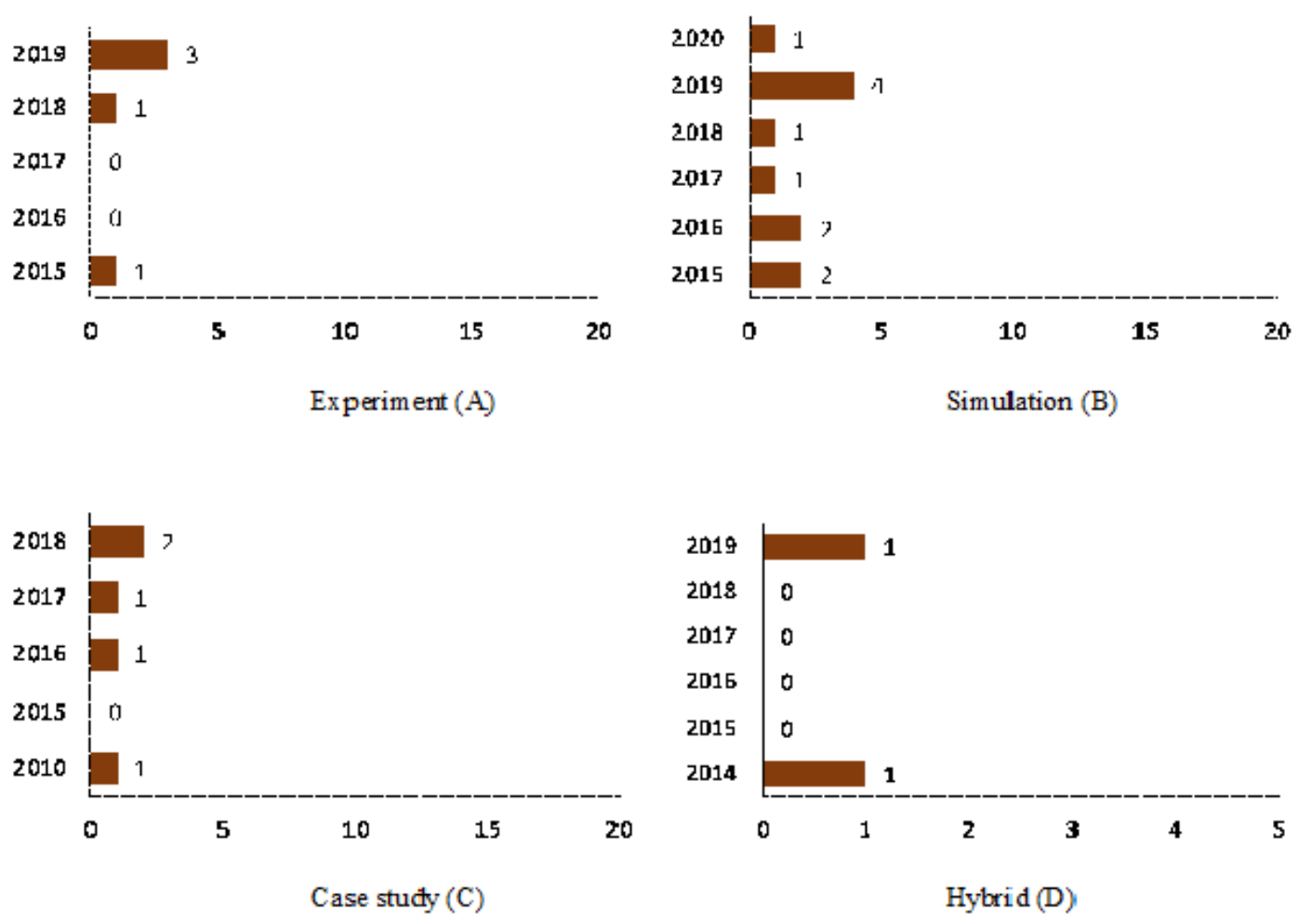

FIGURE 3. (A-D). Breakdowns of evaluation measures used in the respective years of evaluation 
TABLE 1. Analysis of the studies on data collection in loT

\begin{tabular}{|c|c|c|c|c|c|}
\hline Ref & Propsal & Architecture & Use cases & Year & Publication channel \\
\hline [61] & System & $\begin{array}{l}\text { IIOT sensor cloud, IIOT gateway, and } \\
\text { IIOT field device }\end{array}$ & Nil & 2015 & Conference \\
\hline [33] & System & Central server, gateway, and Internet & Agriculture & 2015 & Journal \\
\hline [34] & Framework & Nil & Smart Environment & 2015 & Journal \\
\hline$[4]$ & Framework & IOT, gateway, and network server & Agriculture & 2019 & Journal \\
\hline$[35]$ & System & Central server, gateway, and Internet & Agriculture & 2019 & Journal \\
\hline$[36]$ & System & Sink node, gateway, and Internet & Agriculture & 2015 & Magazine \\
\hline [37] & Model & $\begin{array}{l}\text { Cloud-based IOT platform, gateway, } \\
\text { software defined network, WSN, and } \\
\text { indoor location system }\end{array}$ & Nil & 2016 & Conference \\
\hline [38] & Algorithm & $\begin{array}{l}\text { Network manager, cloud, and cogni- } \\
\text { tive radio sensor network }\end{array}$ & Nil & 2018 & Journal \\
\hline [39] & Protocol & $\begin{array}{l}\text { Cluster gateway, sink node, cluster } \\
\text { head, and cluster members }\end{array}$ & Smart environment & 2020 & Journal \\
\hline$[40]$ & Framework & $\begin{array}{l}\text { IOT cloud, semantic overlay layer, } \\
\text { service virtualization layer, and real } \\
\text { world access layer }\end{array}$ & Mechanical sector & 2010 & Conference \\
\hline$[41]$ & System & $\begin{array}{l}\text { NB-IoT cloud platform, data receiv- } \\
\text { ing and transmission networks, NB- } \\
\text { IoT terminals, and application server }\end{array}$ & Academic research & 2017 & Journal \\
\hline [11] & System & Nil & Nil & 2017 & Conference \\
\hline [42] & Algorithm & Nil & $\begin{array}{l}\text { Smart city environ- } \\
\text { ment }\end{array}$ & 2016 & Journal \\
\hline$[43]$ & Algorithm & Nil & Trade & 2019 & Journal \\
\hline$[44]$ & Framework & $\begin{array}{l}\text { Security testing module, management } \\
\text { and report module, security testing } \\
\text { manager module and measurements, } \\
\text { and analysis module }\end{array}$ & Nil & 2019 & Journal \\
\hline [45] & Nil & Nil & Smart environment & 2016 & Journal \\
\hline [46] & Framework & $\begin{array}{l}\text { Application layer, business layer, } \\
\text { middleware layer, perception layer, } \\
\text { and network layer }\end{array}$ & Software engineering & 2018 & Conference \\
\hline [46] & Nil & Nil & Manufacturing & 2016 & Conference \\
\hline$[62]$ & Nil & Gateway,network domain & Nil & 2016 & Journal \\
\hline [48] & Framework & Nil & $\begin{array}{l}\text { E-science, manufac- } \\
\text { turing and smart cities }\end{array}$ & 2012 & Conference \\
\hline [49] & Framework & Application layer & Smart environment & 2014 & Magazine \\
\hline [50] & Model & $\begin{array}{l}\text { Devices, communication protocol, } \\
\text { bus layer, processing and analytics, } \\
\text { Web }\end{array}$ & Mechanical sector & 2017 & Conference \\
\hline [6] & Framework & $\begin{array}{l}\text { Internet application layer (IAL), local } \\
\text { communication layer, physiological } \\
\text { sensing layer, information processing } \\
\text { layer, and user application layer }\end{array}$ & Health & 2014 & Conference \\
\hline [47] & Framework & Nil & Smart things & 2018 & Conference \\
\hline [7] & Model & $\begin{array}{l}\text { Application layer, service system } \\
\text { layer, physical layer, and IoT gateway }\end{array}$ & $\begin{array}{l}\text { Health and smart city } \\
\text { environment }\end{array}$ & 2018 & Journal \\
\hline [54] & System & $\begin{array}{l}\text { Network, application protocol, and } \\
\text { root }\end{array}$ & Nil & 2014 & Conference \\
\hline$[58]$ & Approach & Nil & Nil & 2019 & Conference \\
\hline [59] & Model & Nil & smart environment & 2019 & Journal \\
\hline [63] & Algorithm & Nil & Nil & 2019 & Journal \\
\hline$[60]$ & Framework & IoT nodes, blockchain, cloud, and fog & Nil & 2019 & Journal \\
\hline
\end{tabular}

\section{1) State-of-the-art}

Piyare et al. introduced a WSN architecture derived from cloud-based sensor data platform open.sen.se [8]in which the architecture display, access, and sharing of the infographic of different data streams from anywhere can be achieved with Internet connectivity . An experiment was conducted and the results were evaluated to assess alert notification time, accessibility, and battery consumption. The results demonstrate improved performances in these aspects. Porambage et al. introduced an authentication mechanism for WSNs in distributed IoT devices [65]. The developed mechanism uses a multiple-phase authentication protocol, which enables the user and sensor nodes to authenticate and establish connections. The experimental results reveal that the protocol is feasible for use in WSNs. Kaiwartya proposed a framework that will provide optimized fault tolerance (FT) during WSN virtualization [66]. The framework focuses on heterogeneous networks for IoT applications. They conducted a case studybased simulation to assess the performance of the framework. Duan et al. used a game approach to propose an energy-aware 
TABLE 2. Performance measures and evaluation metrics with respect to the studies on loT

\begin{tabular}{|c|c|c|c|}
\hline Ref & Performance measures & Evaluation measures & Year of publication \\
\hline [61] & Scalability, security, and performance & Experiment & 2015 \\
\hline [33] & Nil & Nil & 2015 \\
\hline [34] & Latency and performance & Simulation & 2015 \\
\hline [4] & Energy consumption & Experiment & 2019 \\
\hline [35] & Nil & Simulation & 2019 \\
\hline [36] & Network lifetime, energy consumption & Simulation & 2015 \\
\hline$[37]$ & Performance & Simulation & 2016 \\
\hline [38] & Network lifetime, Network performance & Simulation & 2018 \\
\hline [39] & $\begin{array}{l}\text { Throughput, latency and network lifetime, en- } \\
\text { ergy consumption }\end{array}$ & Simulation & 2020 \\
\hline [40] & Lifetime & Case study & 2010 \\
\hline [41] & Nil & Nil & 2017 \\
\hline [11] & Nil & simulation & 2017 \\
\hline$[42]$ & Performance and data transmission & simulation & 2016 \\
\hline [43] & Efficiency & $\begin{array}{l}\text { Simulation and theoretical } \\
\text { analysis }\end{array}$ & 2019 \\
\hline$[44]$ & Security and validity & Simulation & 2019 \\
\hline$[45]$ & Nil & Case study & 2016 \\
\hline [46] & Accuracy, security and privacy, and reliability & Case study & 2018 \\
\hline$[47]$ & Nil & Nil & 2016 \\
\hline [64] & Privacy & Nil & 2016 \\
\hline$[48]$ & Nil & Nil & 2012 \\
\hline [49] & Nil & Nil & 2014 \\
\hline$[50]$ & Nil & Case study & 2017 \\
\hline [6] & Nil & Nil & 2014 \\
\hline [51] & Security, privacy, and network latency & Experiment & 2018 \\
\hline [7] & Nil & Case study & 2018 \\
\hline [54] & Throughput and performance & $\begin{array}{l}\text { Simulation and benchmark } \\
\text { comparison }\end{array}$ & 2014 \\
\hline [58] & $\begin{array}{l}\text { Reliability, security, performance, and data } \\
\text { transmission }\end{array}$ & Experiment & 2019 \\
\hline [59] & $\begin{array}{l}\text { Performance, data transmission, and communi- } \\
\text { cation }\end{array}$ & Simulation & 2019 \\
\hline$[63]$ & $\begin{array}{l}\text { Energy consumption, accuracy, and perfor- } \\
\text { mance }\end{array}$ & Experiment & 2019 \\
\hline [60] & Nil & Simulation & 2019 \\
\hline
\end{tabular}

trust derivation scheme that can be used to manage overhead and maintain adequate WSN security [62]. Their simulation results indicate that the approach not only decreases energy consumption but maintains significant network security. An approach for data dissemination called transmission with multiple load balancing schemes (TMLBSs) has been proposed by Liu et al. [67]. The strategy uses a nature-inspired approach and colony optimization. The authors conducted simulations to validate the approach's performance, and the results proved its superiority and effectiveness in terms of load balance level and network longevity. [68]. conducted a performance investigation on the IoT-enabled intra-vehicular wireless sensor networks (IVWSNs). Meanwhile, Thombre et al. adopted open technologies to WSN stack [69] and simulated the stack using a Contiki network simulator (Cooja) with varying conditions, which helped to understand the performance of the stack and benchmark analysis. Dong et al. proposed a method that collects data on energy efficiency and reliability requirement called reliability and multipath encounter routing (RMER) [1]. The RMER approach provides an improved network lifetime and event detection reliability while reducing energy consumption. The experimental simulation results indicate that the proposed techniques outper- form other solutions in the event detections. [70] proposed a software-defined WSN architecture to manage WSNs, while [71] proposed a lightweight algorithm called adaptive transmission power control (ATPC) in WSN. The algorithm manages the differences in radio communication and low-power sensor devices. The simulation experiments results show that ATPC achieves more significant energy savings and is robust even with environmental based on the timing. Mainetti et al. [3] discussed solutions to integrate heterogeneous WSNs and proposed a harmonized framework that will allow new installations and legacy ones. The proposed framework is currently being tested in the building automation scenarios and provides a logical overlay on sensor networks, which exploits the virtual sensor concept. Lee and Cheng in [72] proposed fuzzy-logic-based clustering approach to improve the WSN lifetime through workload distribution The experimental simulation results revealed that the approach is more efficient than the compared algorithms in terms of network lifetime. Li et al. in [73] introduced a CS-based framework for WSNs and IoT, which offers a stable approach for compressible signal and data in information systems. Guo et al. in [74] proposed opportunistic flooding, which is delay driven flooding method that is developed for low-duty WSNs. 
Their simulation results revealed that the performance of the proposed method is close to the optimal. The proposed approach also achieved short flooding delay compared with improved traditional flooding. Another study adopted realistic simulation models under the many-to-one communication approach to highlight and evaluate techniques to examine how information can be collected from a WSN ordered as a tree [75]. Liu et al. in [76] proposed techniques for data collection schemes for WSNs. The study adopted a powerlaw decaying data model to propose an estimation algorithm. The techniques require lesser zipped measurements, which reduces energy consumption. The evaluation results indicate that the proposed algorithm prolongs the network lifetime compared with existing approaches.Authors in [77] integrate WSNs, the concept of big data, and big data systems to provide an efficient operation of WSNs. They also presented a survey of distinct energy-efficient techniques for handling big data in WSNs. Meanwhile, authors in [2] proposed an efficient architecture that will use the WSN and Bluetooth protocol to improve road travel safety. The experimental results revealed that integrating the sensor and Bluetooth technology improved road travel safety. One study proposed architecture with distributed and hierarchical controllers [78]. Their experimental results revealed that the architecture reduces the influence of software-defined networking control traffic in WSNs and the data flow installation time on the network.authors in [79]introduced an approach-based trustworthy architecture for WSNs and reported that the proposed approach outperforms the existing trust management techniques for WSN. Lounis et al. developed an architecture for accessing and collecting a huge volume of data produced by medical sensor networks [80]. The authors also proposed a flexible and effective security mechanism to outsource the collected medical data. [5] summarized the attacks and their classifications in WSNs and explored the security mechanisms widely used to handle those attacks. Khan et al. [81] proposed a data annotation architecture for semantic applications in virtualized heterogeneous WSNs. They built a prototype that they then deployed in the cloud environment using the Google App engine. The initial performance measurements presented the efficient nature of the proposed architecture. Doddapaneni et al. [82] proposed a framework that would allow developers to model the software architecture of WSNs distinctly. Table 3 highlights the details of the data collection studies on WSNs. Figure 4 shows the respective publication channels identified from the studies on data collection in WSN. For WSN studies, we identified three publication channels, namely, Journal, Conference, and Symposium. Journal has the highest number of studies (14), followed by Conferences(3), and Symposium (1).

\section{2) Performance Measures}

In this section, the performance measures utilized by the respective data collection studies on WSNs are outlined. Table 4 provides detailed information about the performance measures and evaluation measures used by each study.

\section{3) Evaluation measures}

This section identified three evaluation measures used by WSN studies, namely, Experiment adopted by 4 studies, Simulation adopted by 12 studies, and Hybrid adopted by 1 study. Figure 5 highlights each evaluation measure's detailed breakdown with respect to the years of utilization in the respective studies. For Simulation, which is the most utilized by data collection studies on WSNs, 2012 was the most active year with 4 studies utilizing it, followed by 2016 with 3 studies. The trend shows that Simulation's utilization is declining, with no more than one study published each year from 2017 to 2019. This section presents the use cases of data collection in WSNs. Therefore, we identified some use cases, namely, smart environment, flood detection, building automation, transport system, health, and the mechanical sector.

\section{4) Use Cases}

Smart environments Similar to IoT, WSNs were used in smart environments in most of the studies reviewed [8] [65] [72] . These studies utilized WSNs with sensing devices to collect data in smart environments. Hence, with these technologies, useful key areas in a given environment can be understood, environmental planning can be standardized, and important areas in the smart environment can be tracked. Therefore, the data collected in such environments can be very useful, and effective data collection methods are very important. Flood detection and control The studies we reviewed [74] [69] investigated data collection in WSNs. This use case is particularly critical to many countries, as flood detection and control are crucial in saving lives. Hence, with effective data collection mechanisms to collect data in studying the nature of floods, better detection and control methods can be devised and implemented.

\section{5) Others}

Various applications were also recorded in other use cases. For example,authors in [1] applied WSN in event detection and distortion, [3]in building automation, [2] in the transport system, [80] in health, and finally, [81] in a semantic application.

\section{DATA COLLECTION IN SENSOR CLOUD}

This subsection outlines the detailed discussion and analysis of data collection with respect to SC. Facets, such as the state-of-the-art, proposals, performance measures, evaluation measures, and use cases are outlined and discussed.

\section{1) State-of-the-art}

Wang et al.in [83] proposed a mobile edge computing-based intelligent trust evaluation scheme to assess sensor nodes' efficiency by utilizing a probabilistic graphical model. They also proposed a moving algorithm for mobile edge nodes. The experimental evaluation was compared with the traditional scheme. The evaluation results revealed that the proposed scheme decreased energy consumption and effectively 
TABLE 3. Analysis of the data collection studies on WSNs

\begin{tabular}{|c|c|c|c|c|c|}
\hline Ref & Propsal & Architecture & Use cases & Year & Publication channel \\
\hline$[8]$ & System & $\begin{array}{l}\text { Sensor layer, coordinator layer, and } \\
\text { supervision layer }\end{array}$ & Smart environment & 2013 & Conference \\
\hline$[65]$ & Protocol & $\begin{array}{l}\text { IoT Cloud, gateway node, and certifi- } \\
\text { cate authority }\end{array}$ & Smart Environment & 2014 & Conference \\
\hline$[66]$ & Framework & Nil & Nil & 2018 & Journal \\
\hline [62] & Model & Nil & Nil & 2014 & Journal \\
\hline [67] & Approach & Nil & Nil & 2019 & Journal \\
\hline [68] & Architecture & $\begin{array}{l}\text { Information acquisition and informa- } \\
\text { tion processing }\end{array}$ & Nil & 2017 & Journal \\
\hline [69] & Stack & Application, networking, and sensing & $\begin{array}{ll}\text { Flood detection } \\
\text { (smart city) }\end{array}$ & 2016 & Journal \\
\hline$[1]$ & Approach & Nil & $\begin{array}{l}\text { event detection dis- } \\
\text { tortion }\end{array}$ & 2016 & Journal \\
\hline [70] & Architecture & Nil & Nil & 2012 & Journal \\
\hline [71] & Algorithm & Node, packet, and radio range & Nil & 2016 & Journal \\
\hline$[72]$ & Algorithm & Nil & Smart Environment & 2012 & Journal \\
\hline [73] & Framework and algorithm & $\begin{array}{l}\text { Internet network, data acquisition net- } \\
\text { work, and data analysis network }\end{array}$ & Nil & 2013 & Journal \\
\hline [74] & Approach & Nil & Nil & 2014 & Journal \\
\hline [75] & Algorithm & Nil & Nil & 2012 & Journal \\
\hline [76] & Algorithm & Nil & Nil & 2015 & Journal \\
\hline [79] & Architecture & Nil & Nil & 2012 & Journal \\
\hline$[80]$ & Architecture & $\begin{array}{l}\text { Cloud server, WSN, monitoring ap- } \\
\text { plication, and health care authority }\end{array}$ & Health & 2012 & Conference \\
\hline$[81]$ & Architecture & $\begin{array}{l}\text { Non-semantic application, semantic } \\
\text { application, and application overlay } \\
\text { layer }\end{array}$ & Nil & 2015 & Symposium \\
\hline
\end{tabular}

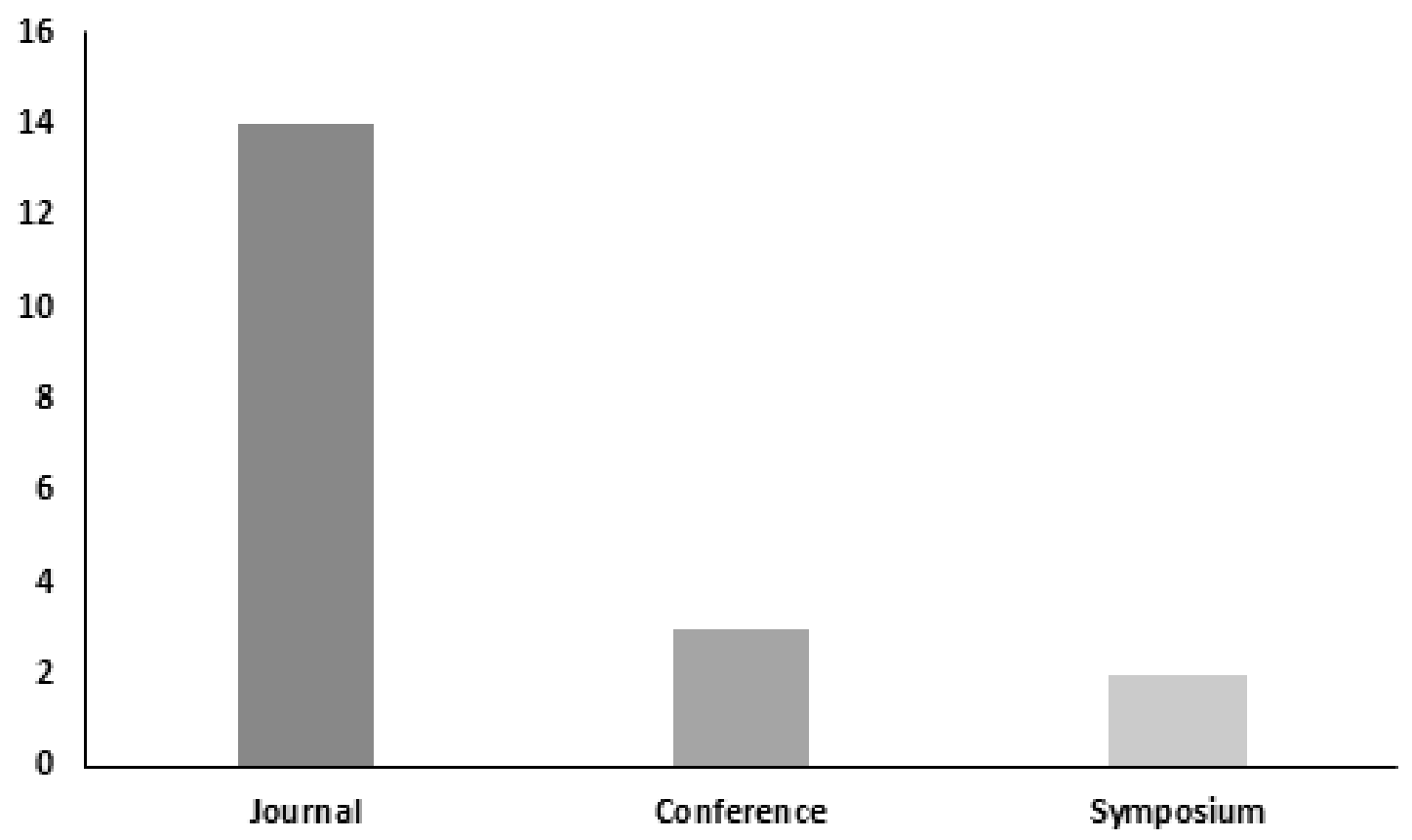

FIGURE 4. Analysis of the publication channels for data collection studies on WSN 
TABLE 4. Performance measures and evaluation metrics with respect to the studies on WSN

\begin{tabular}{|l|l|l|c|}
\hline Ref & Performance measures & Evaluation measures & Year of publication \\
\hline$[8]$ & $\begin{array}{l}\text { Energy consumption, accessibility, and data } \\
\text { transmission }\end{array}$ & Experiment & 2013 \\
\hline$[65]$ & Performance & Experiment & 2014 \\
\hline$[66]$ & Sensor communication & Case study-based Simulation & 2018 \\
\hline$[62]$ & $\begin{array}{l}\text { Performance, energy consumption latency, and } \\
\text { sensor security and efficiency }\end{array}$ & Simulation & 2014 \\
\hline$[67]$ & Data transmission & Simulation & 2019 \\
\hline$[68]$ & Performance and throughput & Simulation & 2017 \\
\hline$[69]$ & Throughput and latency & Simulation & 2016 \\
\hline$[1]$ & $\begin{array}{l}\text { Energy consumption, network lifetime and data } \\
\text { transmission }\end{array}$ & Simulation & 2016 \\
\hline$[70]$ & Network throughput & Simulation & 2012 \\
\hline$[71]$ & Energy consumption and data transmission & Simulation & 2016 \\
\hline$[72]$ & Performance and network lifetime & Simulation & 2012 \\
\hline$[73]$ & Network life time and energy consumption & Simulation & 2013 \\
\hline$[74]$ & Flooding delay and energy consumption & Simulation & 2014 \\
\hline$[75]$ & Network size and performance & Simulation & 2012 \\
\hline$[76]$ & Energy consumption, network lifetime & Simulation & 2015 \\
\hline$[79]$ & $\begin{array}{l}\text { Communication overhead, memory consump- } \\
\text { tion, energy consumption, and performance }\end{array}$ & Simulation & 2012 \\
\hline$[80]$ & Data transmission and security & Experiment & 2012 \\
\hline$[25]$ & Scalability and delay & Experiment & 2015 \\
\hline
\end{tabular}

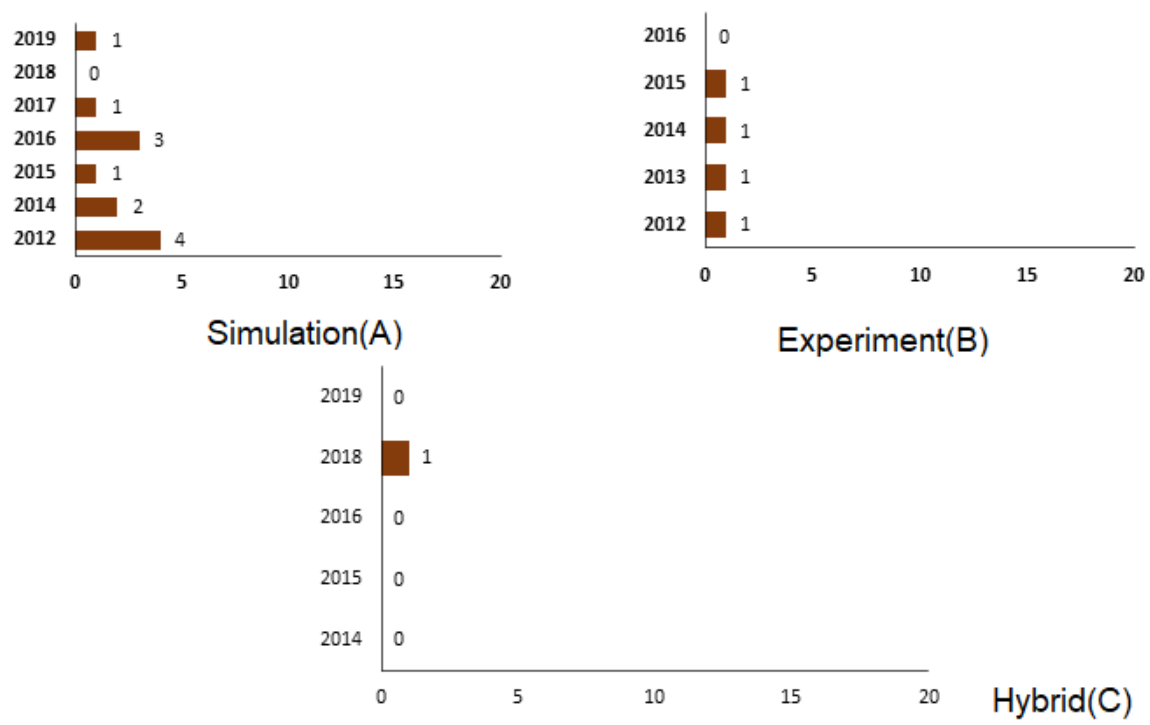

FIGURE 5. (A-C). Breakdowns of evaluation measures used in their respective years

reduced mobile edge nodes' moving distance. Zhu et al. in [84] incorporated five SC pricing models to propose a comprehensive pricing model. The model's analysis shows that it can be very useful for researchers investigating SC pricing mechanisms. To increase the lifetime of WSN, Dinh and Kim in [10] proposed an efficient model for physical sensors and information providers. When the sensor nodes produce sensing information, then the information provided will provide sensing services to the information consumer. The evaluation results revealed that the model improved WSN energy efficiency and service availability compared to the existing models. Zhu et al. Authors in [85] introduced a scheme called multi-method data delivery (MMDD), which incorporated the concept of heterogeneous delivery. The eval- uation results show that MMDD can decrease delivery time and cost for the SC users. Srinivasa et al. detailed the framework PARASENSE architecture, which integrates WSN with IoT [86]. Their results showed that the PARASENSE architecture facilitated the applications' real-time deployment. In another study of Dinh and Kim (2017a), an integrated SC efficient model was proposed to enable the SC to offer sensing services for multiple applications with different latency requirements [9]. The experimental results demonstrate the effective model control sensing of flow latency as compared to existing models. Meanwhile, the study of Misra et al. addressed theoretical characterization and analysis in SC by presenting a mathematical formulation of SC, which will help learn the behavior of WSN-based applications in 
the SC platform [87]. The analysis results show that the SC architecture outperforms WSN by decreasing the energy consumption and increasing the lifetime of the sensor. Zhou et al. developed an approach that integrates IoT and cloud computing [88]. The study analyzed the current state of IoT and cloud integration as well as proposed the cloud things architecture, a cloud-based IoT that houses CloudThings. Zingirian and Valenti (2012) presented a novel SC paradigm for vehicle communication platforms (VCPs) [89]. Based on the paradigm, VCPs will avail all the sensor components, such as vehicle sensors and devices, to third-party vehicle monitoring applications. The study further developed a SC service prototype that supports real-time intelligent truck monitoring (ITM) . The study of Kumar and Madria proposed techniques that will enable the efficient and secure code dissemination in SC due to its focus on WSN by the existing code dissemination techniques [90]. Berrahal et al. proposed a model that depends on incorporating heterogeneous WSN and cloud computing to build distributed public safety databases [91]. The proposed approach is evaluated through a simulation. Huang et al. studied random early detectionbased (RED-based) congestion control for data transmission in SC and proposed an improved RED (IRED) algorithm [92].The results of the experimental simulation show some promise. Iacono et al. Authors in [13] introduced the adoption of standard cloud file synchronization services (CFSS) to manage WSNs in the SC. They experimented using SC to validate the proposal. Liu et al. optimized intrusion detection strategy to reduce energy consumption and decrease alarm messages in SC [93]. The simulation results demonstrate some improvement. Tao et al. [94] introduced a cache network scheme, which is built based on cache nodes in edge networks. They also formulated the data replica placement problem as a mixed-integer programming (MIP) problem to reduce storage, access, and placement costs. Then, they reduced this to a linear programming problem, which is more straightforward to resolve than the original MIP problem. Lawson and Ramaswamy in [95] introduced cloud monitoring service techniques that know the data quality and energy efficiency tradeoff levels. They also developed an optimized energy architecture that enables WSNs to provide an effective data stream and meet consumer data quality needs. Guerreiro et al. proposed a sensor cloud model that suits emerging IoT sensing applications as a business model, and their evaluation results revealed that the best approach for resource allocation depends on the suppliers/consumers scenario. In another study, Guerreiro et al. proposed a resource allocation model to assign sensors and cloud resources to clients [12] . The evaluation results show that the model can incorporate techniques that allocate fewer devices while selecting the adequate ones for application requirements. Ahmed et al.in [45] introduced IoT-based smart environments and focused on the current state, classification, and open research challenges. The identified publication channels with respect to the number of studies published are presented in Figure 6. Table 5 highlights the data collection studies on SC in detail.

\section{2) Performance Measures}

Table 6 highlights the performance and evaluation measures utilized by the respective SS. Among these, performance measures, such as energy consumption and data transmission, are the most utilized measures by studies on SC.

\section{3) Evaluation measures}

Table 6 provides the evaluation measures for each study. For the analysis in this section, we have identified four evaluation measures: Experiment adopted by 6 studies, Simulation adopted by 6 studies, Case study adopted by 2 studies, and Hybrid adopted by 1 study. Figure 7 presents the detailed breakdown of each evaluation measure with respect to the years of utilization in line with the respective studies.

\section{4) Use Cases}

This section presents the use cases of data collection studies on SC. We identified very few studies in this area. Hence, only four use cases were identified. These use cases include [34] in smart surveillance systems, [87] and [84] in business, [89]in intelligent truck monitoring, and [10] in smart environments. As an extension of WSN, SC's application in various domains is important to ensure efficient and effective data collection. We recommend that researchers focus more on data collection using SC in various environments. Table 7 highlights the use of cases in the three domains (IoT, WSN, and SC).

\section{SIMILARITIES AND DIFFERENCES IN DATA COLLECTION BETWEEN IOT, WSN, AND SC}

In this section, the similarities and differences in data collection among IoT, WSN, and SC are discussed. Due to the multifaceted nature of this paper, this section is broken down into different subsections that target specific analyzed factors, such as use cases and architecture. This is done in order to specifically discuss each factor with respect to the similarities or differences related to each factor among respective facets . Table 7 presents the use cases utilized in IoT, WSN, and SC based on the SS in terms of use cases. These three facets' applications and services were adopted in a range of use cases, such as health care, environmental monitoring and industrial task, surveillance, senior residents monitoring, and so on. The three selected domains were all applied in smart environments, as shown in Table 7. The SC and WSN were also applied in weather forecasting and flood detection [74], [31] [69]. The findings show that none of the IoT studies reviewed focused on weather forecasting, environmental monitoring, and disaster detection even though these technologies can be used to efficiently monitor the vibration in a building during an earthquake. Moreover, the three research domains were applied in the smart environment use case to enhance and support the capabilities of its users in executing their tasks. 


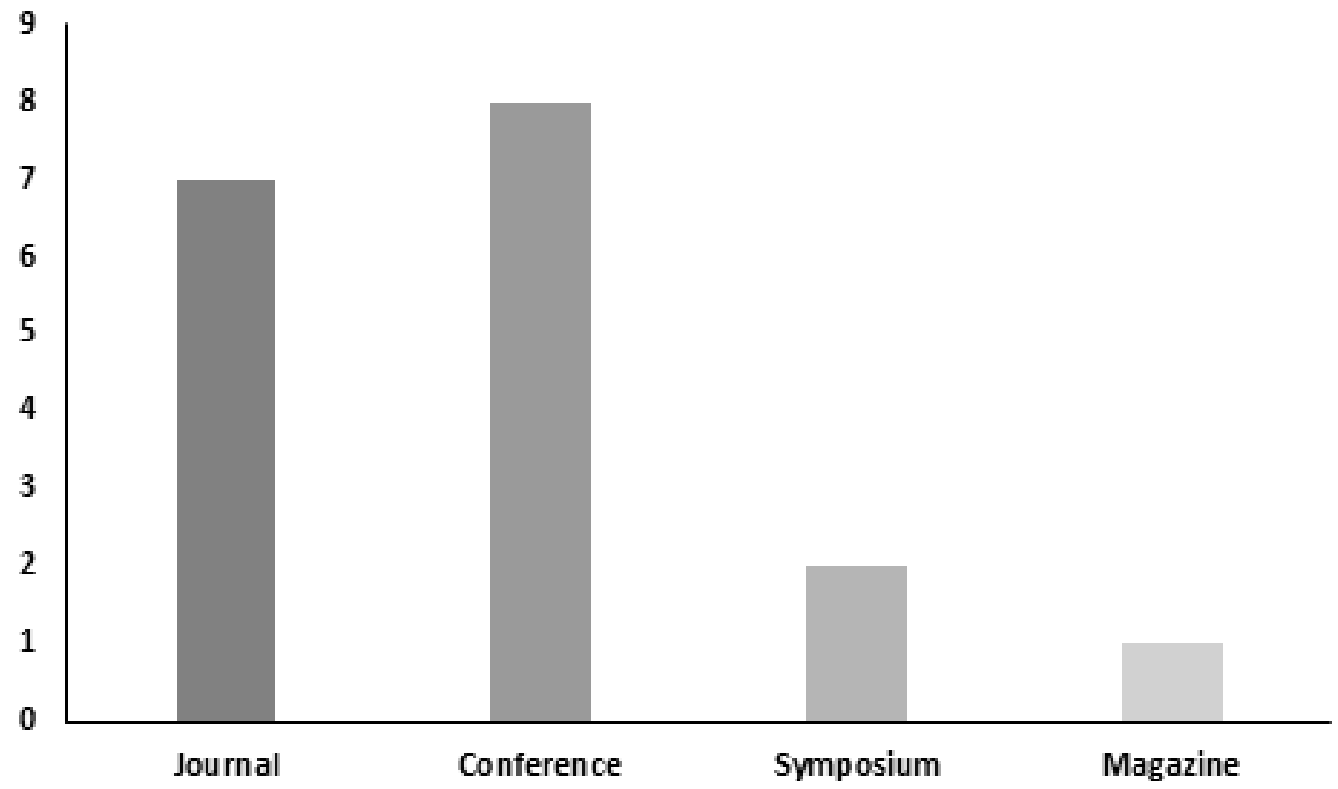

FIGURE 6. Analysis of the publication channels for data collection studies on SC

TABLE 5. Analysis of the data collection studies on SC

\begin{tabular}{|c|c|c|c|c|}
\hline Ref & Proposal & Use Cases & Year of publication & Publication channel \\
\hline$[83]$ & Evaluation Scheme & $\begin{array}{l}\text { Smart } \\
\text { system }\end{array}$ & 2020 & Journal \\
\hline$[84]$ & Model & Business & 2015 & Conference \\
\hline$[10]$ & Model & Nil & 2017 & Conference \\
\hline$[85]$ & Scheme & Nil & 2017 & Magazine \\
\hline [9] & Model & Smart environment & 2017 & Symposium \\
\hline$[87]$ & Model & Business & 2017 & Journal \\
\hline$[88]$ & Approach & Nil & 2013 & Conference \\
\hline [89] & Architecture & Nil & 2012 & Symposium \\
\hline$[90]$ & Approach and algorithm & Nil & 2014 & Conference \\
\hline [91] & Approach & Nil & 2016 & Conference \\
\hline$[92]$ & Algorithm & Nil & 2014 & Journal \\
\hline$[13]$ & Model & Nil & 2017 & Journal \\
\hline$[93]$ & Model & Nil & 2018 & Journal \\
\hline [94] & Algorithm & Nil & 2015 & Journal \\
\hline [95] & Architecture & Nil & 2015 & Conference \\
\hline [12] & Model & Nil & 2019 & Journal \\
\hline$[91]$ & Architecture & Nil & 2016 & Conference \\
\hline
\end{tabular}

\section{A. USE CASES}

The smart environment is one of the use cases utilized by the selected domains, in which sensors are connected through networking devices to collaboratively work and make human lives easier [45]. Similarly, in agriculture, it has been shown that there is no existing study that implemented SC technology. However, a few have applied WSNs and the IoT in their works. While the IoT improves agricultural productivity and efficiency, the WSN serves as a driver of smart agriculture [4] [35] [32] [33]. However, there is an increased need for decision support systems in precision agriculture, which are built using WSN [33]. Precision agriculture provides a wide range of solutions to water scarcity, food shortage, and deterioration of soil properties [4]. Other use cases of the selected domains identified by this study are E-science, manufacturing, and industry. IoT enables the interconnection between the physical world and cyberspace to provide a promising opportunity in manufacturing applications and building powerful services [47]. Moreover, the emergence of industrial IoT results in device manageability and connectivity potential through incorporated architecture [11]. Our analysis results show that only the transport system has been implemented in the WSN use case from the selected domains. In the healthcare system, both IoT and WSN have been adopted, which may be due to the fact that IoT and WSN can be utilized in multiple devices, such as cameras and bed, heat, stove, and accelerometer sensors [52]. Architecture refers to the extensive description that helps detect chal- 
TABLE 6. Performance measures and evaluation metrics with respect to the studies on SC

\begin{tabular}{|l|l|l|c|}
\hline Ref & Performance measures & Evaluation measures & Year of publication \\
\hline$[83]$ & Energy consumption and performance & Simulation & 2020 \\
\hline$[84]$ & Performance and data transmission & Analysis & 2015 \\
\hline$[10]$ & $\begin{array}{l}\text { Temperature and humidity and energy effi- } \\
\text { ciency }\end{array}$ & Nil & 2017 \\
\hline$[85]$ & Data transmission & Case study & 2017 \\
\hline$[9]$ & $\begin{array}{l}\text { Latency, energy consumption, and temperature } \\
\text { and humidity }\end{array}$ & Experiment & 2017 \\
\hline$[87]$ & $\begin{array}{l}\text { Fault tolerance, energy consumption, and life- } \\
\text { time of a sensor node }\end{array}$ & Case study & 2017 \\
\hline$[88]$ & Temperature & Case study & 2013 \\
\hline$[89]$ & Nil & Experiment & 2012 \\
\hline$[90]$ & Data transmission and energy consumption & Simulation & 2014 \\
\hline$[91]$ & Data privacy and availability and reliability & Simulation & 2016 \\
\hline$[92]$ & $\begin{array}{l}\text { Throughput, data transmission, and perfor- } \\
\text { mance }\end{array}$ & Simulation & 2014 \\
\hline$[13]$ & Data transmission and communication & Experiment & 2017 \\
\hline$[93]$ & Energy consumption and data security & Simulation & 2018 \\
\hline$[94]$ & Performance & Experiment & 2015 \\
\hline$[95]$ & Data transmission and energy efficiency & Nil & 2015 \\
\hline$[12]$ & Scalability & Experiment & 2018 \\
\hline$[12]$ & Data transmission & Experiment & 2019 \\
\hline$[91]$ & Nil & Simulation & 2016 \\
\hline
\end{tabular}
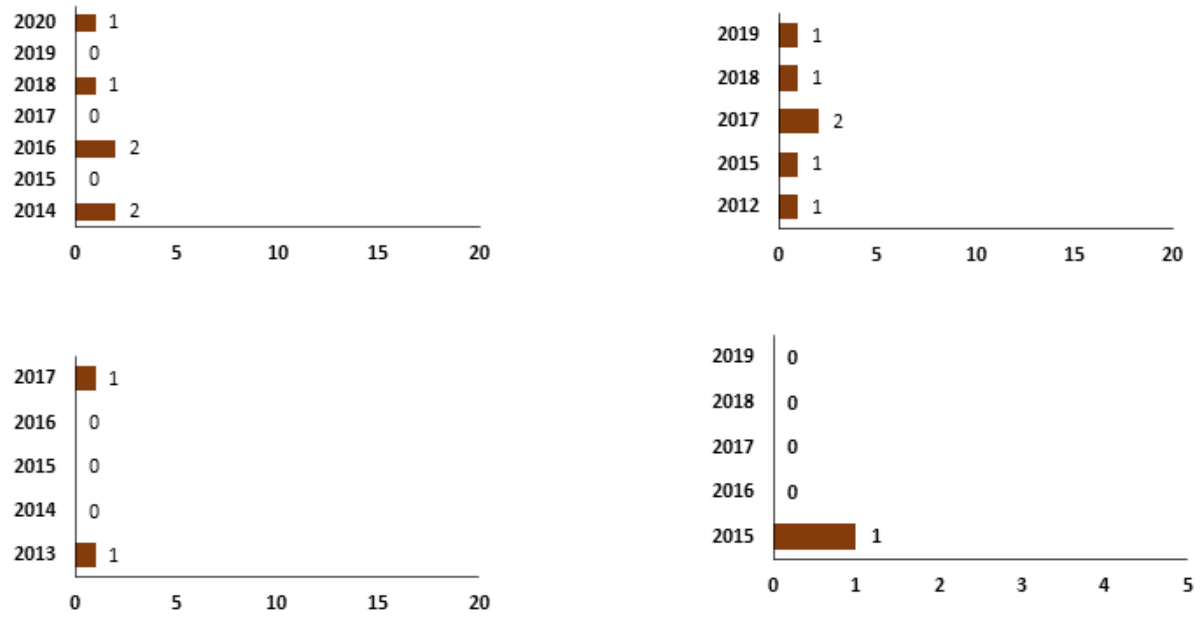

FIGURE 7. $4(\mathrm{~A}-\mathrm{C})$. Breakdowns of evaluation measures used in their respective years

lenges and issues for application scenarios [52]. Different architectures with varying topologies have been used in the three selected domains. The studies reviewed for IoT architecture have utilised various architectural frameworks. Such architectures as cloud-based IoT platforms, software-defined networks, and various novel communication platforms have been proposed and utilized [45] [49] [39] [31] [42] [34]. Blockchain technology framework has also been utilized in this domain for data collection. For WSN, the architectural framework is mainly the same, consisting of sensor nodes and based stations with distinct data collection routing mechanisms. The application often gets altered if customized applications are used in other use cases [8] [65] [72]. The aim is to connect and improve data collection efficiency in
WSN framework. The SC architecture framework comprises a WSN framework and cloud computing. Hence, SC is the combination of these two technologies. Therefore, WSN and $\mathrm{SC}$ architectural frameworks are similar. From the reviewed studies in SC, virtual sensors, fog computing, SC databases, and Internet protocol (IP) module are generally utilized in the architecture [84] [34] [87] [89] [9] . Hence, data collection is done from sensor devices to the cloud. We conclude that WSN architecture is more similar to SC architecture than IoT architecture. Therefore, IoT architecture is less similar to the two technologies (WSN and SC). Concerning the performance measures and evaluation measures, they are generally similar for the studies reviewed in the three domains. Performance measures, such as throughput, energy 
TABLE 7. Use cases in three selected domains

\begin{tabular}{|c|c|c|c|}
\hline Use Cases & IoT & WsN & SC \\
\hline Agriculture & $\checkmark$ & $\checkmark$ & X \\
\hline Smart surveillance system & X & X & $\checkmark$ \\
\hline Building automation & X & $\checkmark$ & X \\
\hline Smart environment & $\checkmark$ & $\checkmark$ & $\checkmark$ \\
\hline Mechanical sector & $\checkmark$ & X & X \\
\hline Event distortion detection & X & $\checkmark$ & X \\
\hline Academic research & $\checkmark$ & X & X \\
\hline Business/trade & $\checkmark$ & X & $\checkmark$ \\
\hline Software engineering & $\checkmark$ & X & X \\
\hline Flood detection & X & $\checkmark$ & $\checkmark$ \\
\hline E-science & $\checkmark$ & X & X \\
\hline Healthcare & $\checkmark$ & $\checkmark$ & X \\
\hline Transport system & X & $\checkmark$ & X \\
\hline Semantic application & X & $\checkmark$ & X \\
\hline Weather forecasting & X & $\checkmark$ & \\
\hline Intelligent truck monitoring & X & X & $\checkmark$ \\
\hline Mathematical analysis & $\checkmark$ & X & X \\
\hline
\end{tabular}

consumption, latency, and network lifetime, are all used in all the domains' respective studies. Likewise, evaluation measures like simulation, experiment, and case study are similar. Hence, with respect to these facets (performance measures and evaluation measures), the three domains are similar.

\section{OPEN RESEARCH CHALLENGES}

This section highlights the research challenges identified from the previous sections' results. It further provides the direction for future works, which will aid both new and veteran researchers in the selected domains.

\section{A. SCALABILITY}

This is the first challenge identified in this study. The term "scalability" refers to the capability of a network or system to handle the rising scale of any environment. The study of Zhou et al. incorporated cloud computing and IoT to propose an architecture that conveniently allows scalable network access to shared computing resources [88]. Hence, scalability with respect to data collection has proven to be challenging in IoT and WSNs works. However, this challenge has been resolved by the introduction of data collection strategies using SC. Nevertheless, scalability is still a key concern in the respective research domains. Our findings indicate that the novel SC technologies improve the scalability and storage capacity and enhance the efficiency of packet delivery.

\section{B. PACKET ROUTING}

Another challenge identified by this study is how the routing packet can be transmitted between the packet source and its destination without delay and congestion. In all the domains, packet routing is a key issue because it determines the data transmission speed and time during data collection. Hence, an efficient routing protocol that effectively and proficiently transmits data from source to destination in IoT, WSN, and $\mathrm{SC}$ is still needed.

\section{FLEXIBILITY}

Based on our analysis, flexibility has been identified as a concerning challenge, particularly in WSN. The study of [8] highlighted the flexibility issues and centralized decisionmaking process in WSN. The authors further designed and implemented a more flexible architecture that integrates WSN to cloud to improve flexibility and decision-making capability. However, flexibility is still a growing concern.

\section{VIRTUALIZATION}

The results also revealed that virtualization is another challenge in the selected domains. Virtualization refers to the ability of multiple devices in IoT, WSN, and SC to share hardware resources. The virtualization process also allows multiple software applications to run on the same server by creating more than one virtual machine. The study of [66] addressed communication failure in WSN environments with the aid of IoT virtualization. Similarly, [60] developed an IoT virtualization framework based on the sensor as a service notion, which comprised of three layers: the semantic layer, real-world layer, and virtualization layer for IoT virtualization. The framework can provide efficient IoT virtualization. However, despite the authors' effort, efficient virtualization mechanisms and frameworks are still needed, particularly in $\mathrm{SC}$ architecture.

\section{E. COMMUNICATION}

Another issue identified in the selected domains is communication. There is an absence of a typical platform that conceals the heterogeneity of communication technologies and offers a straightforward naming service to different applications. The obvious communication heterogeneity issue is resource sharing in heterogeneous sensor networks [59]. Furthermore, the sensor nodes in a WSN might be shared by various applications with disparate objectives. In this manner, with an expansion in the utilization of WSNs, there is a need to create mechanisms that can proficiently serve numerous applications simultaneously. In an existing study, WSNs were used to develop a cooperative communications technique to provide efficient battery usage in WSNs and energy savings [19]. The cooperative communications technique can improve the WSNs' reliability. Hence, more works on developing new techniques that will address communication issues are needed.

\section{F. DEVICE MANAGEMENT}

IoT, WSN, and SC device management is a challenging issue that has to be addressed. The study of [11]. used Ethereum and blockchain to develop a framework to manage IoT devices. [79] proposed an approach-based trustworthy architecture for WSN, which deliberates on the system's challenges and focuses on the collaborative mechanisms for trust evaluation, maintenance, and management techniques for WSNs [79]. Another study proposed an architecture that will address the issue of data management in WSNs by accessing and collecting a huge volume of data produced by 
medical sensor networks [80]. Therefore, an efficient device management architecture in IoT, WSN, and SC during data collection is optimal.

\section{CONCLUSION}

This study presented a comprehensive survey that investigates the data collection methods employed in studies regarding three selected domains: WSN, IoT, and SC. The survey was conducted by searching and categorizing all existing and available studies in the selected domains. Only the relevant papers were selected during the search and selection process of the thousands of papers identified in the first database search. The finding of this study is set to equip the research community with detailed descriptions of data collection and the similarities and differences among the three selected domains. In terms of the publication source, we found that conference papers occupy a larger proportion of the papers used in this study, followed by journal articles, symposiums, and magazines. Furthermore, our analysis showed the existence of nine contributions in the three selected domains, namely, framework, algorithm, model, protocol, approach, method, architecture, system, and topology. However, it is observed that model, algorithm, and framework are the most proposed contributions in the selected domains. Additionally, five evaluation mechanisms were identified to be utilized by the SS: experiment, case study, simulation, theoretical analysis, and comparative analysis. Moreover, the experimental simulation was adopted by most of the studies in the domains selected. Finally, this study highlighted the research challenges and future research directions for new and veteran researchers in this field.

\section{REFERENCES}

[1] M. Dong, K. Ota, and A. Liu, "Rmer: Reliable and energy-efficient data collection for large-scale wireless sensor networks," IEEE Internet of Things Journal, vol. 3, no. 4, pp. 511-519, 2016.

[2] V. Katiyar, P. Kumar, and N. Chand, "An intelligent transportation systems architecture using wireless sensor networks," International Journal of Computer Applications, vol. 14, no. 2, pp. 22-26, 2011.

[3] L. Mainetti, L. Patrono, and A. Vilei, "Evolution of wireless sensor networks towards the internet of things: A survey," in SoftCOM 2011, 19th international conference on software, telecommunications and computer networks. IEEE, 2011, pp. 1-6.

[4] X. Feng, F. Yan, and X. Liu, "Study of wireless communication technologies on internet of things for precision agriculture," Wireless Personal Communications, vol. 108, no. 3, pp. 1785-1802, 2019.

[5] V. Kumar, A. Jain, P. Barwal et al., "Wireless sensor networks: security issues, challenges and solutions," International Journal of Information and Computation Technology (IJICT), vol. 4, no. 8, pp. 859-868, 2014.

[6] P. P. Ray, "Home health hub internet of things (h 3 iot): An architectural framework for monitoring health of elderly people," in 2014 International Conference on Science Engineering and Management Research (ICSEMR). IEEE, 2014, pp. 1-3.

[7] P. Chamoso, A. González-Briones, S. Rodríguez, and J. M. Corchado, "Tendencies of technologies and platforms in smart cities: a state-of-theart review," Wireless Communications and Mobile Computing, vol. 2018, 2018.

[8] R. Piyare, S. Park, S. Y. Maeng, S. H. Park, S. C. Oh, S. G. Choi, H. S. Choi, and S. R. Lee, "Integrating wireless sensor network into cloud services for real-time data collection," in 2013 International Conference on ICT Convergence (ICTC). IEEE, 2013, pp. 752-756.
[9] T. Dinh and Y. Kim, "An efficient sensor-cloud interactive model for on-demand latency requirement guarantee," in 2017 IEEE International Conference on Communications (ICC). IEEE, 2017, pp. 1-6.

[10] —, "Information centric sensor-cloud integration: An efficient model to improve wireless sensor networks' lifetime," in 2017 IEEE International Conference on Communications (ICC). IEEE, 2017, pp. 1-6.

[11] S. Huh, S. Cho, and S. Kim, "Managing iot devices using blockchain platform," in 2017 19th international conference on advanced communication technology (ICACT). IEEE, 2017, pp. 464-467.

[12] J. Guerreiro, L. Rodrigues, and N. Correia, "Modeling of sensor clouds under the sensing as a service paradigm," in International Conference on Broadband Communications, Networks and Systems. Springer, 2018, pp. 22-30.

[13] L. Iacono, C. García Garino, O. Marianetti, and C. Párraga, "Wsns data and configuration management in sensor clouds with cloud file synchronization services," Journal of Computer Science \& Technology, vol. 17, 2017.

[14] L. Da Xu, W. He, and S. Li, "Internet of things in industries: A survey," IEEE Transactions on industrial informatics, vol. 10, no. 4, pp. 2233-2243, 2014.

[15] M. u Farooq, M. Waseem, S. Mazhar, A. Khairi, and T. Kamal, "A review on internet of things (iot)," International Journal of Computer Applications, vol. 113, no. 1, pp. 1-7, 2015.

[16] G. Fortino, L. Fotia, F. Messina, D. Rosaci, and G. M. Sarné, "Trust and reputation in the internet of things: state-of-the-art and research challenges," IEEE Access, vol. 8, pp. 60 117-60 125, 2020.

[17] M. A. Ferrag, M. Derdour, M. Mukherjee, A. Derhab, L. Maglaras, and H. Janicke, "Blockchain technologies for the internet of things: Research issues and challenges," IEEE Internet of Things Journal, vol. 6, no. 2, pp. 2188-2204, 2018.

[18] N. C. Luong, D. T. Hoang, P. Wang, D. Niyato, D. I. Kim, and Z. Han, "Data collection and wireless communication in internet of things (iot) using economic analysis and pricing models: A survey," IEEE Communications Surveys \& Tutorials, vol. 18, no. 4, pp. 2546-2590, 2016.

[19] P. Rawat, K. D. Singh, H. Chaouchi, and J. M. Bonnin, "Wireless sensor networks: a survey on recent developments and potential synergies," The Journal of supercomputing, vol. 68, no. 1, pp. 1-48, 2014

[20] N. A. Pantazis, S. A. Nikolidakis, and D. D. Vergados, "Energy-efficient routing protocols in wireless sensor networks: A survey," IEEE Communications surveys \& tutorials, vol. 15, no. 2, pp. 551-591, 2012.

[21] L. M. Oliveira and J. J. Rodrigues, "Wireless sensor networks: A survey on environmental monitoring." JCM, vol. 6, no. 2, pp. 143-151, 2011.

[22] Y. Yang, L. Wu, G. Yin, L. Li, and H. Zhao, "A survey on security and privacy issues in internet-of-things," IEEE Internet of Things Journal, vol. 4, no. 5, pp. 1250-1258, 2017.

[23] V. J. Hodge, S. O'Keefe, M. Weeks, and A. Moulds, "Wireless sensor networks for condition monitoring in the railway industry: A survey," IEEE Transactions on intelligent transportation systems, vol. 16, no. 3, pp. 10881106,2014

[24] H. Das, B. Naik, B. Pati, and C. R. Panigrahi, "A survey on virtual sensor networks framework," International Journal of Grid Distribution Computing, vol. 7, no. 5, pp. 121-130, 2014.

[25] M. Di Francesco, S. K. Das, and G. Anastasi, "Data collection in wireless sensor networks with mobile elements: A survey," ACM Transactions on Sensor Networks (TOSN), vol. 8, no. 1, pp. 1-31, 2011.

[26] H. Yetgin, K. T. K. Cheung, M. El-Hajjar, and L. H. Hanzo, "A survey of network lifetime maximization techniques in wireless sensor networks," IEEE Communications Surveys \& Tutorials, vol. 19, no. 2, pp. 828-854, 2017.

[27] A. B. Noel, A. Abdaoui, T. Elfouly, M. H. Ahmed, A. Badawy, and M. S Shehata, "Structural health monitoring using wireless sensor networks: A comprehensive survey," IEEE Communications Surveys \& Tutorials, vol. 19, no. 3, pp. 1403-1423, 2017.

[28] A. Alamri, W. S. Ansari, M. M. Hassan, M. S. Hossain, A. Alelaiwi, and M. A. Hossain, "A survey on sensor-cloud: architecture, applications, and approaches," International Journal of Distributed Sensor Networks, vol. 9, no. 2, p. 917923, 2013.

[29] S. K. Dash, S. Mohapatra, and P. K. Pattnaik, "A survey on applications of wireless sensor network using cloud computing," International Journal of Computer Science \& Emerging Technologies, vol. 1, no. 4, pp. 50-55, 2010.

[30] R. K. Dwivedi, M. Saran, and R. Kumar, "A survey on security over sensorcloud," in 2019 9th International Conference on Cloud Computing, Data Science \& Engineering (Confluence). IEEE, 2019, pp. 31-37. 
TABLE 8. Definitions of all acronyms mentioned in the paper

\begin{tabular}{|c|c|}
\hline & Abbreviations \\
\hline IoT & Internet of Things \\
\hline $\mathrm{DC}$ & Data Collection \\
\hline WSNs & Wireless Sensor Networks \\
\hline $\mathrm{SC}$ & Sensor Cloud \\
\hline SS & Selected Studies \\
\hline SHM & Structural Health Monitoring \\
\hline SDN & Software Defined Networking \\
\hline CoAP & Constrained Application Protocol \\
\hline CRSNs & Cognitive Radio Sensor Networks \\
\hline TBEERP & Trust-Based Energy-Efficient Routing Protocol \\
\hline UAU & Unmanned Aerial Vehicles \\
\hline Het-IoT & Heterogeneous IoT \\
\hline NOMA & Nonorthogonal Multiple Access \\
\hline MORA & Multi-Objective Resource Allocation \\
\hline IVWANs & IoT-enabled intra-Vehicular Wireless Sensor Networks \\
\hline RMER & Reliability and Multipath Encounter Routing \\
\hline ATPC & Adaptive Transmission Power Control \\
\hline VCPs & Vehicle Communication Platforms \\
\hline ITM & Intelligent Truck Monitoring \\
\hline RED & Random Early Detection-based \\
\hline IRED & Improved RED \\
\hline CFSS & Cloud File Synchronization Services \\
\hline MIP & Mixed-Integer Programming \\
\hline MMDD & Multimethod Data Delivery \\
\hline IP & Internet protocol \\
\hline
\end{tabular}

[31] Y. Mohammad and S. Nakadai, "Optimal value of information based elicitation during negotiation," in Proceedings of the 18th International Conference on Autonomous Agents and MultiAgent Systems, 2019, pp. 242-250.

[32] B. P. Rao, P. Saluia, N. Sharma, A. Mittal, and S. V. Sharma, "Cloud computing for internet of things \& sensing based applications," in 2012 Sixth International Conference on Sensing Technology (ICST). IEEE, 2012, pp. 374-380.

[33] O. Savale, A. Managave, D. Ambekar, and S. Sathe, "Internet of things in precision agriculture using wireless sensor networks," International Journal of Advanced Engineering \& Innovative Technology, vol. 2, no. 3, pp. 1-5, 2015.

[34] Z. Sheng, H. Wang, C. Yin, X. Hu, S. Yang, and V. C. Leung, "Lightweight management of resource-constrained sensor devices in internet of things," IEEE internet of things journal, vol. 2, no. 5, pp. 402-411, 2015.

[35] K. A. Kumar and K. Ramudu, "Precision agriculture using internet of things and wireless sensor networks," Precision Agriculture, vol. 7, no. 03, p. 5, 2019.

[36] P. Kamalinejad, C. Mahapatra, Z. Sheng, S. Mirabbasi, V. C. Leung, and Y. L. Guan, "Wireless energy harvesting for the internet of things," IEEE Communications Magazine, vol. 53, no. 6, pp. 102-108, 2015.

[37] R. Kirichek, A. Vladyko, M. Zakharov, and A. Koucheryavy, "Model networks for internet of things and sdn," in 2016 18th International Conference on Advanced Communication Technology (ICACT). IEEE, 2016, pp. 76-79.

[38] S. Aslam, W. Ejaz, and M. Ibnkahla, "Energy and spectral efficient cognitive radio sensor networks for internet of things," IEEE Internet of Things Journal, vol. 5, no. 4, pp. 3220-3233, 2018.

[39] M. Ilyas, Z. Ullah, F. A. Khan, M. H. Chaudary, M. S. A. Malik, Z. Zaheer, and H. U. R. Durrani, "Trust-based energy-efficient routing protocol for internet of things-based sensor networks," International Journal of Distributed Sensor Networks, vol. 16, no. 10, p. 1550147720964358, 2020.

[40] S. Alam, M. M. Chowdhury, and J. Noll, "Senaas: An event-driven sensor virtualization approach for internet of things cloud," in 2010 IEEE International Conference on Networked Embedded Systems for Enterprise Applications. IEEE, 2010, pp. 1-6.

[41] J. Chen, K. Hu, Q. Wang, Y. Sun, Z. Shi, and S. He, "Narrowband internet of things: Implementations and applications," IEEE Internet of Things Journal, vol. 4, no. 6, pp. 2309-2314, 2017.

[42] A. Orsino, G. Araniti, L. Militano, J. Alonso-Zarate, A. Molinaro, and A. Iera, "Energy efficient iot data collection in smart cities exploiting d2d communications," Sensors, vol. 16, no. 6, p. 836, 2016
[43] Z. Li, Z. Yang, and S. Xie, "Computing resource trading for edge-cloudassisted internet of things," IEEE Transactions on Industrial Informatics, vol. 15, no. 6, pp. 3661-3669, 2019.

[44] S. Siboni, V. Sachidananda, Y. Meidan, M. Bohadana, Y. Mathov, S. Bhairav, A. Shabtai, and Y. Elovici, "Security testbed for internet-ofthings devices," IEEE transactions on reliability, vol. 68 , no. 1, pp. 23-44, 2019.

[45] E. Ahmed, I. Yaqoob, A. Gani, M. Imran, and M. Guizani, "Internet-ofthings-based smart environments: state of the art, taxonomy, and open research challenges," IEEE Wireless Communications, vol. 23, no. 5, pp 10-16, 2016.

[46] L. Antao, R. Pinto, J. Reis, and G. Gonçalves, "Requirements for testing and validating the industrial internet of things," in 2018 IEEE Internationa Conference on Software Testing, Verification and Validation Workshops (ICSTW). IEEE, 2018, pp. 110-115.

[47] C. Yang, W. Shen, and X. Wang, "Applications of internet of things in manufacturing," in 2016 IEEE 20th International Conference on Computer Supported Cooperative Work in Design (CSCWD). IEEE, 2016, pp. 670 675.

[48] J. Soldatos, M. Serrano, and M. Hauswirth, "Convergence of utility computing with the internet-of-things," in 2012 Sixth International Conference on Innovative Mobile and Internet Services in Ubiquitous Computing. IEEE, 2012, pp. 874-879.

[49] L. Atzori, A. Iera, and G. Morabito, "From" smart objects" to" socia objects": The next evolutionary step of the internet of things," IEEE Communications Magazine, vol. 52, no. 1, pp. 97-105, 2014.

[50] A. Zimmermann, R. Schmidt, K. Sandkuhl, D. Jugel, J. Bogner, and M. Möhring, "Decision-controlled digitization architecture for internet of things and microservices," in International Conference on Intelligent Decision Technologies. Springer, 2017, pp. 82-92.

[51] C. Brooks, C. Jerad, H. Kim, E. A. Lee, M. Lohstroh, V. Nouvelletz, B. Osyk, and M. Weber, "A component architecture for the internet of things," Proceedings of the IEEE, vol. 106, no. 9, pp. 1527-1542, 2018.

[52] E. Sisinni, A. Saifullah, S. Han, U. Jennehag, and M. Gidlund, "Industria internet of things: Challenges, opportunities, and directions," IEEE Transactions on Industrial Informatics, vol. 14, no. 11, pp. 4724-4734, 2018.

[53] Y. Qu, S. Yu, W. Zhou, S. Peng, G. Wang, and K. Xiao, "Privacy of things: Emerging challenges and opportunities in wireless internet of things,' IEEE Wireless Communications, vol. 25, no. 6, pp. 91-97, 2018.

[54] M. Kovatsch, M. Lanter, and Z. Shelby, "Californium: Scalable cloud services for the internet of things with coap," in 2014 International Conference on the Internet of Things (IOT). IEEE, 2014, pp. 1-6. 
[55] W. Wang, S. De, R. Toenjes, E. Reetz, and K. Moessner, "A comprehensive ontology for knowledge representation in the internet of things," in 2012 IEEE 11th International Conference on Trust, Security and Privacy in Computing and Communications. IEEE, 2012, pp. 1793-1798.

[56] D. G. Korzun, A. M. Kashevnik, S. I. Balandin, and A. V. Smirnov, "The smart-m3 platform: Experience of smart space application development for internet of things," in Internet of Things, Smart Spaces, and Next Generation Networks and Systems. Springer, 2015, pp. 56-67.

[57] Y. Liu, K. Wang, Y. Lin, and W. Xu, "A lightweight blockchain system for industrial internet of things," IEEE Transactions on Industrial Informatics, vol. 15, no. 6, pp. 3571-3581, 2019.

[58] W. Liang, M. Tang, J. Long, X. Peng, J. Xu, and K.-C. Li, "A secure fabric blockchain-based data transmission technique for industrial internet-ofthings," IEEE Transactions on Industrial Informatics, vol. 15, no. 6, pp. 3582-3592, 2019.

[59] M. Liu, J. Yang, and G. Gui, "Dsf-noma: Uav-assisted emergency communication technology in a heterogeneous internet of things," IEEE Internet of Things Journal, vol. 6, no. 3, pp. 5508-5519, 2019.

[60] T. Alam, "Design a blockchain-based middleware layer in the internet of things architecture," JOIV: International Journal on Informatics Visualization, vol. 4, no. 1, pp. 28-31, 2020.

[61] P. Hu, "A system architecture for software-defined industrial internet of things," in 2015 IEEE International Conference on Ubiquitous Wireless Broadband (ICUWB). IEEE, 2015, pp. 1-5.

[62] J. Duan, D. Gao, D. Yang, C. H. Foh, and H.-H. Chen, "An energy-aware trust derivation scheme with game theoretic approach in wireless sensor networks for iot applications," IEEE Internet of Things Journal, vol. 1, no. 1, pp. 58-69, 2014.

[63] Y. Chen, N. Zhang, Y. Zhang, X. Chen, W. Wu, and X. S. Shen, "Energy efficient dynamic offloading in mobile edge computing for internet of things," IEEE Transactions on Cloud Computing, 2019.

[64] M. R. Palattella, M. Dohler, A. Grieco, G. Rizzo, J. Torsner, T. Engel, and L. Ladid, "Internet of things in the $5 \mathrm{~g}$ era: Enablers, architecture, and business models," IEEE Journal on Selected Areas in Communications, vol. 34, no. 3, pp. 510-527, 2016.

[65] P. Porambage, C. Schmitt, P. Kumar, A. Gurtov, and M. Ylianttila, "Twophase authentication protocol for wireless sensor networks in distributed iot applications," in 2014 IEEE Wireless Communications and Networking Conference (WCNC). Ieee, 2014, pp. 2728-2733.

[66] O. Kaiwartya, A. H. Abdullah, Y. Cao, J. Lloret, S. Kumar, R. R. Shah, M. Prasad, and S. Prakash, "Virtualization in wireless sensor networks: Fault tolerant embedding for internet of things," IEEE Internet of Things Journal, vol. 5, no. 2, pp. 571-580, 2017.

[67] X. Liu, T. Qiu, and T. Wang, "Load-balanced data dissemination for wireless sensor networks: A nature-inspired approach," IEEE Internet of Things Journal, vol. 6, no. 6, pp. 9256-9265, 2019.

[68] M. A. Rahman, J. Ali, M. N. Kabir, and S. Azad, "A performance investigation on iot enabled intra-vehicular wireless sensor networks," International Journal of Automotive and Mechanical Engineering, vol. 14, pp. 3970-3984, 2017.

[69] S. Thombre, R. U. Islam, K. Andersson, and M. S. Hossain, "Ip based wireless sensor networks: performance analysis using simulations and experiments," Journal of Wireless Mobile Networks, Ubiquitous Computing, and Dependable Applications, vol. 7, no. 3, pp. 53-76, 2016.

[70] T. Luo, H.-P. Tan, and T. Q. Quek, "Sensor openflow: Enabling softwaredefined wireless sensor networks," IEEE Communications letters, vol. 16, no. 11, pp. 1896-1899, 2012.

[71] S. Lin, F. Miao, J. Zhang, G. Zhou, L. Gu, T. He, J. A. Stankovic, S. Son, and G. J. Pappas, "Atpc: adaptive transmission power control for wireless sensor networks," ACM Transactions on Sensor Networks (TOSN), vol. 12, no. 1, pp. 1-31, 2016.

[72] J.-S. Lee and W.-L. Cheng, "Fuzzy-logic-based clustering approach for wireless sensor networks using energy predication," IEEE Sensors Journal, vol. 12, no. 9, pp. 2891-2897, 2012.

[73] S. Li, L. Da Xu, and X. Wang, "Compressed sensing signal and data acquisition in wireless sensor networks and internet of things," IEEE Transactions on Industrial Informatics, vol. 9, no. 4, pp. 2177-2186, 2012.

[74] S. Guo, L. He, Y. Gu, B. Jiang, and T. He, "Opportunistic flooding in low-duty-cycle wireless sensor networks with unreliable links," IEEE Transactions on Computers, vol. 63, no. 11, pp. 2787-2802, 2013.

[75] O. D. Incel, A. Ghosh, B. Krishnamachari, and K. Chintalapudi, "Fast data collection in tree-based wireless sensor networks," IEEE Transactions on Mobile computing, vol. 11, no. 1, pp. 86-99, 2011.
[76] X.-Y. Liu, Y. Zhu, L. Kong, C. Liu, Y. Gu, A. V. Vasilakos, and M.-Y. Wu, "Cdc: Compressive data collection for wireless sensor networks," IEEE Transactions on Parallel and Distributed Systems, vol. 26, no. 8, pp. 2188 2197, 2014

[77] Q. Jawhar, K. Thakur, and K. J. Singh, "Recent advances in handling big data for wireless sensor networks," IEEE Potentials, vol. 39, no. 6, pp. 22-27, 2020.

[78] B. T. de Oliveira and C. B. Margi, "Distributed control plane architecture for software-defined wireless sensor networks," in 2016 IEEE International Symposium on Consumer Electronics (ISCE). IEEE, 2016, pp. 85-86.

[79] V. S. Dhulipala, N. Karthik, and R. Chandrasekaran, "A novel heuristic approach based trust worthy architecture for wireless sensor networks," Wireless personal communications, vol. 70, no. 1, pp. 189-205, 2013.

[80] A. Lounis, A. Hadjidj, A. Bouabdallah, and Y. Challal, "Secure and scalable cloud-based architecture for e-health wireless sensor networks," in 2012 21st International Conference on Computer Communications and Networks (ICCCN). IEEE, 2012, pp. 1-7.

[81] I. Khan, R. Jafrin, F. Z. Errounda, R. Glitho, N. Crespi, M. Morrow, and P. Polakos, "A data annotation architecture for semantic applications in virtualized wireless sensor networks," in 2015 IFIP/IEEE International Symposium on Integrated Network Management (IM). IEEE, 2015, pp. 27-35.

[82] K. Doddapaneni, E. Ever, O. Gemikonakli, I. Malavolta, L. Mostarda, and H. Muccini, "A model-driven engineering framework for architecting and analysing wireless sensor networks," in 2012 Third International Workshop on Software Engineering for Sensor Network Applications (SESENA). IEEE, 2012, pp. 1-7.

[83] T. Wang, H. Luo, W. Jia, A. Liu, and M. Xie, "Mtes: An intelligent trust evaluation scheme in sensor-cloud-enabled industrial internet of things," IEEE Transactions on Industrial Informatics, vol. 16, no. 3, pp. 2054 2062, 2019

[84] C. Zhu, V. C. Leung, E. C.-H. Ngai, L. T. Yang, L. Shu, and X. Li, "Pricing models for sensor-cloud," in 2015 IEEE 7th International Conference on Cloud Computing Technology and Science (CloudCom). IEEE, 2015, pp. 454-457.

[85] C. Zhu, V. C. Leung, K. Wang, L. T. Yang, and Y. Zhang, "Multi-method data delivery for green sensor-cloud," IEEE Communications Magazine, vol. 55 , no. 5, pp. 176-182, 2017.

[86] K. Srinivasa, N. Siddiqui, and A. Kumar, "Parasense-a sensor integrated cloud based internet of things prototype for real time monitoring applications," in 2015 IEEE Region 10 Symposium. IEEE, 2015, pp. 53-57.

[87] S. Misra, S. Chatterjee, and M. S. Obaidat, "On theoretical modeling of sensor cloud: A paradigm shift from wireless sensor network," IEEE Systems journal, vol. 11, no. 2, pp. 1084-1093, 2014.

[88] J. Zhou, T. Leppanen, E. Harjula, M. Ylianttila, T. Ojala, C. Yu, H. Jin, and L. T. Yang, "Cloudthings: A common architecture for integrating the internet of things with cloud computing," in Proceedings of the 2013 IEEE 17th international conference on computer supported cooperative work in design (CSCWD). IEEE, 2013, pp. 651-657.

[89] N. Zingirian and C. Valenti, "Sensor clouds for intelligent truck monitoring," in 2012 IEEE Intelligent Vehicles Symposium. IEEE, 2012, pp. 999-1004.

[90] V. Kumar and S. Madria, "Efficient and secure code dissemination in sensor clouds," in 2014 IEEE 15th International Conference on Mobile Data Management, vol. 1. IEEE, 2014, pp. 103-112.

[91] S. Berrahal, N. Boudriga, and A. Bagula, "Cooperative sensor-clouds for public safety services in infrastructure-less areas," in 2016 22nd AsiaPacific Conference on Communications (APCC). IEEE, 2016, pp. 222229.

[92] J. Huang, D. Du, Q. Duan, Y. Zhang, Y. Zhao, H. Luo, Z. Mai, and Q. Liu, "Modeling and analysis on congestion control for data transmission in sensor clouds," International Journal of Distributed Sensor Networks, vol. 10, no. 3, p. 453983, 2014.

[93] J. Liu, J. Yu, and S. Shen, "Energy-efficient two-layer cooperative defense scheme to secure sensor-clouds," IEEE Transactions on Information Forensics and Security, vol. 13, no. 2, pp. 408-420, 2017.

[94] Y. Tao, Y. Zhang, and Y. Ji, "Efficient data replica placement for sensor clouds," IET Communications, vol. 10, no. 16, pp. 2162-2169, 2016.

[95] V. Lawson and L. Ramaswamy, "Data quality and energy management tradeoffs in sensor service clouds," in 2015 IEEE International Congress on Big Data. IEEE, 2015, pp. 749-752. 


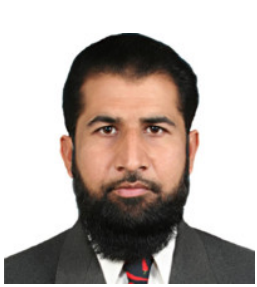

IHSAN ALI (M'2015-SM'2020) is currently working as a Research Associate at the Faculty of Computer Science and Information Technology, University of Malaya. He has published more than 40 high impact research journal papers including, highly reputable journals, IEEE communications surveys and tutorials, Communication Magazine with more than 170 cumulative Impact factor.He is actively involved in research teaching activities for the last 10 years in different countries, including Saudi Arabia, USA, Pakistan, and Malaysia. He is also member of the editorial board of different journals, including Wireless Communications and Mobile Computing and Journal of Computer Networks and Communications. He is lead GE of Special issue Data Collection in ResourceLimited Networks (WSNs, IoT, Sensor Cloud) and have served as a technical program Committee Member for several well-known conferences including IWCMC 2017-2018, AINIS 2017, Future 5V 2017, ICACCI-2018, INAIT 2019, DiCES-N19, CCNC2020, ICCAIS2020 and CSNT2020 and also an organizer of the Special session on fog computing in Future 5V 2017. He also participated in and organized several conferences and workshops in different countries. He is an active reviewer for several high repute journal including Computers electrical engineering, KSII Transactions on Internet and Information Systems, Mobile Networks and Applications, International Journal of Distributed sensor networks, Journal of Advanced Transportation, IEEE Transactions on Intelligent Transportation Systems, Computer Networks, IEEE Access, wireless communications, and Mobile Computing and IEEE Communication Magazine. My research interests include Wireless Sensor Networks, Cloud Computing, Sensor Cloud, Fog Computing, IoT, and Underwater Sensor Network 